\title{
Assessing the Impact of the Tropopause on Mountain Waves and Orographic Precipitation Using Linear Theory and Numerical Simulations
}

\author{
Nicholas Siler AND DALE DURRAN \\ Department of Atmospheric Sciences, University of Washington, Seattle, Washington
}

(Manuscript received 14 July 2014, in final form 30 September 2014)

\begin{abstract}
The partial reflection of mountain waves at the tropopause has been studied extensively for its contribution to downslope windstorms, but its impact on orographic precipitation has not been addressed. Here linear theory and numerical simulations are used to investigate how the tropopause affects the vertical structure of mountain waves and, in turn, orographic precipitation. Relative to the no-tropopause case, wave-induced ascent above the windward slope of a two-dimensional ridge is found to be enhanced or diminished depending on the ratio of the tropopause height to the vertical wavelength of the mountain waves-defined here as the "nondimensional tropopause height" $\hat{H}$. In idealized simulations of flow over both two-dimensional and three-dimensional ridges, variations in $\hat{H}$ are found to modulate the precipitation rate by roughly a factor of 2 under typical atmospheric conditions. The sensitivity of precipitation to $\hat{H}$ is related primarily to the depth of windward ascent but also to the location and strength of leeside descent, with significant impacts on the distribution of precipitation across the range (i.e., the rain-shadow effect). Using a modified version of Smith and Barstad's orographic precipitation model, variations in $\hat{H}$ are found to produce significant rain-shadow variability in the Washington Cascades, perhaps explaining some of the variability in rain-shadow strength observed among Cascade storms.
\end{abstract}

\section{Introduction}

The amount and spatial distribution of orographic precipitation depend to a large degree on how the topography modifies the airflow above it (e.g., Rotunno and Houze 2007). For example, convective precipitation can occur over windward slopes when conditionally unstable air is forced upward by the topography (e.g., Kirshbaum and Durran 2004; Medina et al. 2010). Conversely, when stable air encounters a tall topographic barrier, the flow can become "blocked," causing ascent and precipitation to shift upstream (e.g., Houze et al. 2001; Medina and Houze 2003; Jiang 2003; Colle 2004). In many cases, however, convection and blocking are minimal and the airflow is approximately linear, with patterns of ascent and precipitation determined primarily by terrain-induced internal gravity waves (i.e., "mountain waves") (e.g., Smith 1979; Garvert et al. 2007; Siler et al. 2013).

Corresponding author address: Nicholas Siler, Department of Atmospheric Sciences, University of Washington, Box 351640, Seattle, WA 98195.

E-mail: siler@atmos.washington.edu
According to linear theory, the behavior of mountain waves is governed by the vertical structure of winds and static stability (e.g., Queney 1960). At the interface of vertical layers with different wind speeds and/or static stabilities, mountain waves can be partially reflected, significantly altering the flow field (Eliassen and Palm 1961). Yet while this effect has been studied extensively in the context of downslope wind storms, (e.g., Klemp and Lilly 1975; Lilly 1978; Durran 1986), its impact on orographic precipitation has largely been overlooked. One notable exception is the recent paper by Barstad and Schüller (2011), in which the authors used an extension of the linear model of Smith and Barstad (2004, hereafter SB04) to simulate orographic precipitation in the presence of two tropospheric layers with different wind speeds and microphysical time scales. However, this study did not consider how orographic precipitation is affected by the abrupt change in stability at the tropopause, which is well known to cause mountain-wave reflection (e.g., Klemp and Lilly 1975; Worthington and Thomas 1997).

In this paper, therefore, we consider how orographic precipitation is affected by the reflection of mountain waves at the tropopause. We begin in section 2 with 
a two-dimensional linear analysis of ascent above the windward slope of a two-dimensional sinusoidal ridge. We find that low-level ascent is either enhanced or diminished depending on the ratio of the tropopause height to the vertical wavelength of the mountain waves. We then approximate the precipitation generated by linear mountain waves over an isolated ridge and investigate how this responds to changes in wind speed, tropospheric stability, and tropopause height. In section 3, we perform a series of numerical simulations using a nonhydrostatic, nonlinear model, the results of which are found to be broadly consistent with our linear analysis from section 2. In section 4, we use our own modified version of the SB04 model to illustrate how wave reflection by the tropopause may account for some of the variability in precipitation patterns observed among storms in the Washington Cascades. We conclude with a summary and discussion of our results in section 5 .

\section{Linear theory}

\section{a. The effect of a tropopause on windward ascent}

We begin with a linear analysis of the vertical velocity field for a single Fourier component in the $x-z$ plane,

$$
w(x, z)=\hat{w}(z) e^{i k x},
$$

for which the surface topography consists of an infinite series of sinusoidal ridges of the form

$$
h(x)=h_{0} e^{i k x} .
$$

Using the Boussinesq approximation, the linear, inviscid, steady-state vertical velocity field is governed by the one-dimensional Helmholtz equation,

$$
\frac{d^{2} \hat{w}}{d z^{2}}+l^{2} \hat{w}=0
$$

where $l^{2}$ is the so-called Scorer parameter, defined as

$$
l^{2}=\frac{N^{2}}{U^{2}}-\frac{1}{U} \frac{d^{2} U}{d z^{2}}-k^{2},
$$

with $U(z)$ representing the speed of the background flow and $N(z)$ the Brunt-Väisälä frequency.

Here we focus on the case of a two-layer atmosphere, consisting of a troposphere with constant static stability $N$, capped by an infinitely deep stratosphere with constant $N_{s}$. For now we assume that $U$ is constant throughout both layers. Applying the lower boundary condition,

$$
w(x, 0)=U \frac{d h}{d x},
$$

along with the conventional radiation and matching conditions (e.g., Klemp and Lilly 1975), the solution to (3) within the troposphere is found to be

$$
\hat{w}(z)=i U k h_{0}\left(C^{+} e^{i m z}+C^{-} e^{-i m z}\right),
$$

where

$$
m=\left(\frac{N^{2}}{U^{2}}-k^{2}\right)^{1 / 2}
$$

is the tropospheric vertical wavenumber, and the constants $C^{ \pm}$are given by

$$
C^{ \pm}=\frac{(\epsilon \pm 1) e^{\mp i m H}}{(\epsilon+1) e^{-i m H}+(\epsilon-1) e^{i m H}}
$$

In (8), $H$ is the height of the tropopause, and

$$
\epsilon=m / m_{s},
$$

or the ratio of wavenumbers across the tropopause. Assuming $k>0$, the terms $\exp (i m z)$ and $\exp (-i m z)$ in (6) represent upward- and downward-propagating waves with a vertical wavelength of

$$
\lambda_{z}=\frac{2 \pi}{m}
$$

Because our primary interest is orographic precipitation, for now we focus on the vertical structure of $w$ over the windward slope, where the bulk of condensation occurs. For the terrain profile described by (2), the steepest windward slope is located at $x_{u}=3 \pi / 2 k$, above which, from (1) and (6),

$$
w\left(x_{u}, z\right)=A\left[\cos m z-\frac{\left(1-\epsilon^{2}\right) \sin 4 \pi \hat{H}}{2\left(\epsilon^{2} \cos ^{2} 2 \pi \hat{H}+\sin ^{2} 2 \pi \hat{H}\right)} \sin m z\right],
$$

where $A=U k h_{0}$ is the wave amplitude in the absence of a tropopause (i.e., $\epsilon=1$ ), and $\hat{H}$ is a nondimensional tropopause height, defined as

$$
\hat{H}=\frac{H}{\lambda_{z}} .
$$

The impact of the tropopause on windward ascent is represented by the second term inside the parentheses in (11). In the no-tropopause limit $\epsilon \rightarrow 1, w\left(x_{u}, z\right)$ behaves like $\cos (m z)$, with ascent at the surface giving way to descent above a quarter vertical wavelength $\left(z=\lambda_{z} / 4\right)$. However, when $\epsilon \neq 1$, the solution includes an additional $\sin m z$ term, the sign of which is determined by the value of $\hat{H}$. At low levels $\left[0<z\left(\lambda_{z} / 4\right)\right]$ important for orographic 
precipitation, $\sin m z$ and $\cos m z$ have the same sign. Therefore, ascent is enhanced in this layer if $\sin 4 \pi \hat{H}<0$, or equivalently, if $n-(1 / 2)<2 \hat{H}<n$, where $n$ is a positive integer. From (12), this condition is met when the tropopause height is a bit less than an integer multiple of a half vertical wavelength. On the other hand, low-level ascent is diminished when $n<2 \hat{H}<n+(1 / 2)$ - that is, when the tropopause is a bit higher than an integer multiple of a half vertical wavelength.

\section{b. Physical interpretation}

To develop some intuition for the physics behind (11), it is instructive to think of the troposphere as a kind of Fabry-Pérot interferometer, with $w\left(x_{u}, z\right)$ representing the interference pattern from an infinite series of waves trapped between the surface (which acts as a total reflector) and the tropopause (which acts as a partial reflector). At the tropopause, reflection is governed by the physical requirement that $\hat{w}$ and $d \hat{w} / d z$ match across the interface $^{1}$ (Eliassen and Palm 1961). For an upwardpropagating wave $e^{i m z}$, these matching conditions take the form

$$
\left.\begin{array}{c}
e^{i m z}+r e^{-i m z}=t e^{i m_{s} z} \\
m e^{i m z}-m r e^{-i m z}=m_{s} t e^{i m_{s} z}
\end{array}\right\} \quad \text { at } \quad z=H
$$

where $r$ and $t$ are coefficients representing the amplitudes of the reflected and transmitted waves. Solving these equations for $r$ yields

$$
r=-r_{0} e^{i 4 \pi \hat{H}}
$$

where

$$
r_{0}=\frac{1-\epsilon}{1+\epsilon}
$$

This implies that any incident wave at the tropopause is related to its reflection by

$$
\overbrace{e^{i m z}}^{\text {upward }} \rightarrow \overbrace{-r_{0} e^{-i(m z-4 \pi \hat{H})}}^{\text {downward }}
$$

Imposing the same conditions for surface reflections (where $t=0$ ) gives $r=-1$, implying that incident and reflected waves are governed by

$$
\overbrace{e^{-i m z}}^{\text {downward }} \rightarrow \overbrace{-e^{i m z}}^{\text {upward }} .
$$

\footnotetext{
${ }^{1}$ Pressure must match at the interface. In our case, $U$ and $d U / d z$ match across the interface, so the linearized $x$-momentum equation implies that $d \hat{w} / d z$ also matches.
}

Applying (16) and (17) iteratively to an initial terraininduced wave of the form $A \exp (i m z)$, the resulting interference pattern is found to be

$w\left(x_{u}, z\right)=A\left[e^{i m z}+\sum_{n=1}^{\infty}\left(r_{0} e^{i 4 \pi \hat{H}}\right)^{n}\left(e^{i m z}-e^{-i m z}\right)\right]$,

which is equivalent to (11), but in the form of an infinite geometric series of upward- and downward-propagating waves.

To understand the consequences of (18), let us consider two specific cases in detail: $\hat{H}=7 / 8$, which represents the midpoint of an interval over which low-level ascent is enhanced, and $\hat{H}=9 / 8$, which represents the midpoint of an interval over which low-level ascent is diminished. The contributions to $w\left(x_{u}, z\right)$ from the first several reflected waves are shown for both cases in Fig. 1.

The case $\hat{H}=7 / 8$ is shown in Fig. 1a. The wave with the largest amplitude, $A \cos m z$, represents the terraininduced wave (i.e., wave 1). According to (16), the partial reflection of this wave at the tropopause results in a downward-propagating wave, $r_{0} A \sin (m z)$, that enhances low-level ascent (wave 2). Wave 2 is in turn reflected by the surface, producing another wave equal to $r_{0} A \sin (m z)$ that enhances ascent even further (wave 3 ). The partial reflection of wave 3 produces a downward-propagating wave equal to $r_{0}^{2} A \cos m z$ (wave 4), but this wave is offset by its reflection (wave 5), which is equal in amplitude but $180^{\circ}$ out of phase from wave 4.

Continuing this line of reasoning, a pattern of alternating sines and cosines emerges, whereby each downwardpropagating wave of the form $\cos (m z)$ is canceled by its reflection, while each downward-propagating wave of the form $\sin (m z)$ is reinforced by its reflection. The coefficients of the $\sin (m z)$ terms form a geometric series,

$$
2 \sum_{n=0}^{\infty}(-1)^{n+1} r_{0}^{2 n+1}
$$

which sum to give

$$
w\left(x_{u}, z\right)=A\left(\cos m z+\frac{2 r_{0}}{1+r_{0}^{2}} \sin m z\right) .
$$

Combining (15) and (20) yields

$$
w\left(x_{u}, z\right)=A\left(\cos m z+\frac{1-\epsilon^{2}}{1+\epsilon^{2}} \sin m z\right),
$$

which is the same result given by (11).

Repeating this exercise for the $\hat{H}=9 / 8$ case (Fig. 1b), we find that each reflected wave of the form $\sin m z$ is $180^{\circ}$ out of phase with its counterpart in the $\hat{H}=7 / 8$ case. Thus, 

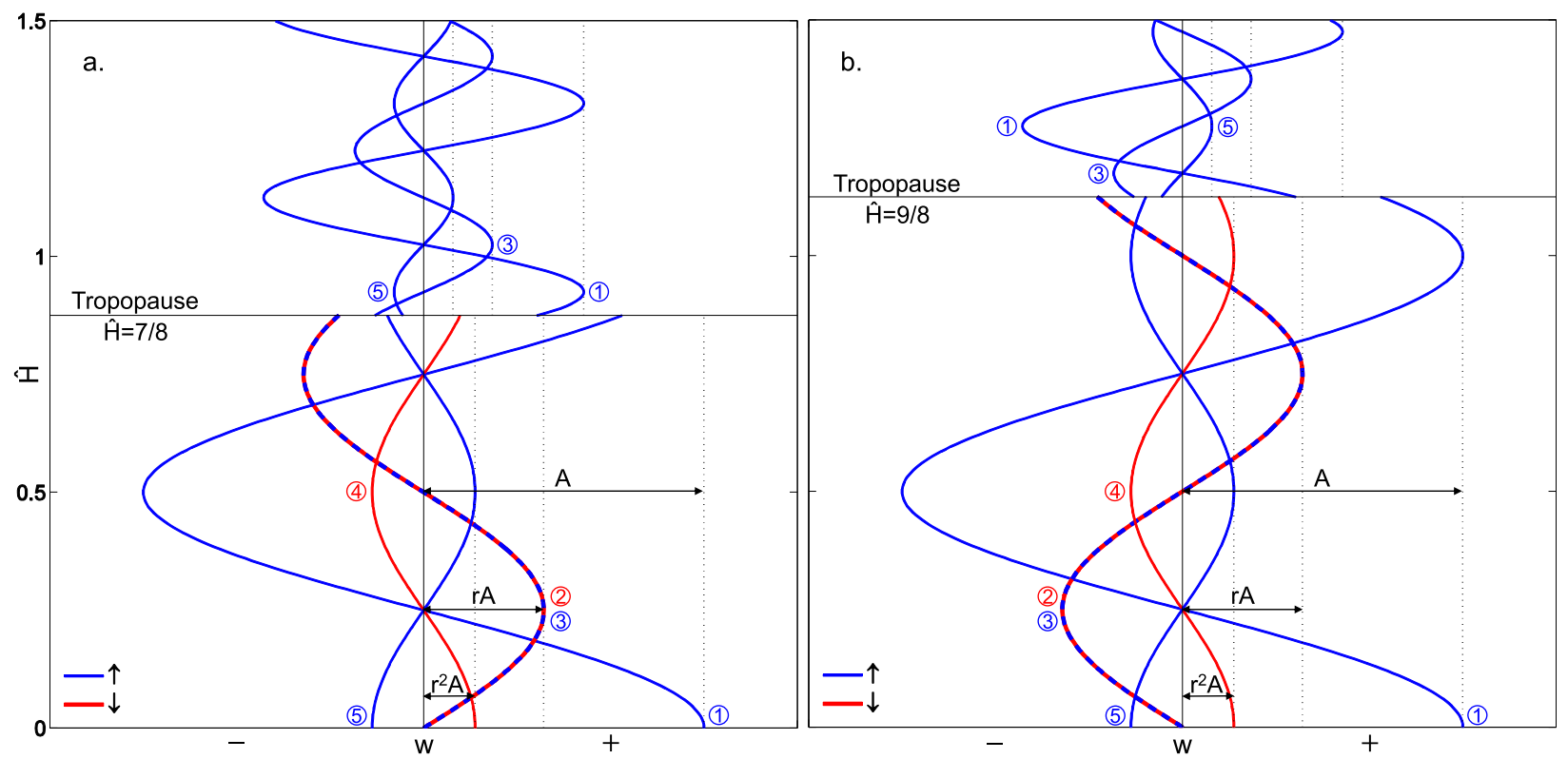

FIG. 1. Contributions to $w\left(x_{u}, z\right)$ from upward- (blue) and downward- (red) propagating waves given (a) $\hat{H}=7 / 8$ and (b) $\hat{H}=9 / 8$. Within the troposphere, partial reflection by the tropopause and total reflection by the surface result in an infinite series of waves, of which only those with amplitude $r_{0}^{2} A$ or greater are shown. The order of waves in the series is indicated by the circled numbers, beginning with the terrain-induced wave, $A \cos (m z)$. Waves 2 and 3 reinforce each other, enhancing low-level ascent in (a) and diminishing it in (b). Waves 4 and 5 offset each other, and their net contribution to $w\left(x_{u}, z\right)$ is therefore zero. Stratospheric waves result from the partial transmission of upward-propagating tropospheric waves across the tropopause.

whereas the initial reflected wave is equal to $r_{0} A \sin (m z)$ in the previous case, here its sign is reversed, implying a reduction in low-level ascent. Following the same procedure as in the previous case, we find that

$$
w\left(x_{u}, z\right)=A\left(\cos m z-\frac{1-\epsilon^{2}}{1+\epsilon^{2}} \sin m z\right)
$$

In the examples above, the impact of $\hat{H}$ on windward ascent has a straightforward physical interpretation, since in both cases the initial reflected wave takes the form $\pm \sin m z$. However, the general case is not fundamentally different. For an arbitrary tropopause height, the initial reflected wave is given by (16) as $-r_{0} A \exp [-i(m z-4 \pi \hat{H})]$, which can be decomposed into two (real) parts: $-\cos 4 \pi \hat{H} \cos m z$ and $-\sin 4 \pi \hat{H} \sin m z$. The first of these terms is proportional to $\cos m z$, and is therefore canceled by its reflection from the surface. However, the second term, which is proportional to $\sin m z$, enhances low-level ascent when $\sin 4 \pi \hat{H}<0$, and weakens it when $\sin 4 \pi \hat{H}>0$, just as in (11). Thus, even when the initial reflected wave is not of the form $\sin (m z)$, it contains a $\sin (m z)$ component that alters low-level ascent just the same.

It is therefore clear that whether $w\left(x_{u}, z\right)$ is enhanced or diminished near the surface depends only on how the initial terrain-induced wave, $A \cos (m z)$, is reflected by the tropopause. When the tropopause height is a bit less than an integer multiple of a half vertical wavelength, as in the $\hat{H}=7 / 8$ case, the reflected wave contains a $+\sin m z$ component, which acts to enhance low-level ascent. On the other hand, when the tropopause height is a bit greater than an integer multiple of a half vertical wavelength, as in the $\hat{H}=9 / 8$ case, the reflected wave contains a $-\sin m z$ component, which acts to diminish low-level ascent.

\section{c. The effect of a tropopause on precipitation}

Thus far we have used linear theory to investigate how the presence of a tropopause affects windward ascent for a single Fourier component. Motivating this analysis was an implicit assumption that the depth and magnitude of windward ascent are fundamentally related to the amount of orographic precipitation. Here we explore this connection more tangibly, using a few simple assumptions to approximate the total precipitation rate from saturated flow over an idealized two-dimensional ridge. The goal of this exercise is to develop a qualitative understanding of the ways in which changes in wind speed, stability, and tropopause height affect orographic precipitation, which will prove helpful for interpreting the numerical results presented in the next section.

To incorporate precipitation within a linear framework, it is necessary to account for the effects of latent 
heating from condensation. One simple way of doing this (e.g., Rotunno and Ferretti 2001; SB04) is to replace $N$ with an "effective static stability," $N^{\prime}$, which we assume to be constant within the troposphere. Since in reality the distribution of latent heating is not uniform within the troposphere, it is perhaps best to think of $N^{\prime}$ as a free parameter, tuned to achieve the best agreement between linear theory and observations/numerical simulations. Nevertheless, previous studies provide some indication of the factors that determine $N^{\prime}$. If the troposphere were saturated everywhere, we might expect $N^{\prime}$ to be close to the troposphere-mean moist Brunt-Väisälä frequency,

$$
\bar{N}_{m}=\frac{1}{H} \int_{0}^{H} N_{m}(z) d z
$$

with $N_{m}$ given by Lalas and Einaudi (1973) as

$$
N_{m}^{2}=\frac{g}{T}\left(\frac{d T}{d z}+\Gamma_{m}\right)\left(1+\frac{L q}{R T}\right)-\frac{g}{1+q} \frac{d q}{d z},
$$

where $g$ is the acceleration of gravity, $T(z)$ is temperature, $\Gamma_{m}(z)$ is the moist adiabatic lapse rate, $L$ is the latent heat of vaporization, $q(z)$ is the mixing ratio of water vapor, and $R$ is the specific gas constant for dry air. In reality, descending regions in the lee are often subsaturated, resulting in an abrupt increase in stability from $N_{m}$ to $N$ as a parcel passes over the ridge (e.g., Barcilon and Fitzjarrald 1985). However, idealized simulations performed by Jiang (2003) suggest that subsaturated regions may not have much effect on $N^{\prime}$, implying that $N^{\prime} \approx \bar{N}_{m}$ may still hold. In the next section, we present numerical results that are broadly consistent with the notion that $N^{\prime} \approx \bar{N}_{m}$, except when the lower troposphere approaches convective instability (Fig. 7b).

We begin our precipitation analysis with linear theory as before, assuming a two-layer atmosphere with constant $U$ and a saturated troposphere, flowing over an isolated "witch-of-Agnesi" ridge of the form

$$
h(x)=\frac{h_{0} a^{2}}{a^{2}+x^{2}},
$$

where $h_{0}$ is the ridge height and $a$ is the half-width. In this case, $w(x, z)$ can be found within the troposphere by replacing $h_{0}$ in (6) with the Fourier transform of (25),

$$
\hat{h}(k)=\frac{h_{0} a}{2} e^{-|k| a},
$$

and taking the inverse transform, given by

$$
w(x, z)=i U h_{0} a \int_{0}^{\infty}\left(C^{+} e^{i m z}+C^{-} e^{-i m z}\right) k e^{-k a} e^{i k x} d k .
$$

In general, a closed-form expression for $w(x, z)$ does not exist owing to the dependence of $m$ on the horizontal wavenumber $k$ [see (7)]. However, if the ridge is broad enough that $N^{\prime} a / U \gg 1$, the dominant wavenumbers in (27) satisfy the hydrostatic condition, $k^{2} \ll N^{\prime 2} / U^{2}$, and the solution within the troposphere can be approximated as

$$
w(x, z)=-\frac{U h_{0} a}{\left(a^{2}+x^{2}\right)^{2}}\left[2 a x \cos m z+\frac{\epsilon\left(a^{2}-x^{2}\right)-a x\left(1-\epsilon^{2}\right) \sin 4 \pi \hat{H}}{\epsilon^{2} \cos ^{2} 2 \pi \hat{H}+\sin ^{2} 2 \pi \hat{H}} \sin m z\right],
$$

where

$$
m=N^{\prime} / U
$$

is the hydrostatic vertical wavenumber,

$$
\hat{H}=\frac{1}{2 \pi} \frac{H N^{\prime}}{U}
$$

is the hydrostatic nondimensional tropopause height, and $^{2}$

\footnotetext{
${ }^{2} N^{\prime}$ determines the phase shift between the ground and the tropopause and is, therefore, a deep-layer quantity. In contrast, $\epsilon$ determines the strength of reflection due to the change in stability at the tropopause and is, therefore, a local quantity. Since $N_{m} \approx N$ in the upper troposphere, where moisture is scarce, $\epsilon \approx N / N_{s}$, not $N^{\prime} / N_{s}$. This approximation is further supported by the numerical simulations presented in the next section.
}

$$
\epsilon \approx \frac{N}{N_{s}}
$$

As proof of the relevance of our earlier analysis to the present isolated ridge, note that at the point $x=-a$, which lies one-half width upwind of the crest, (28) simplifies to

$w(-a, z)=\frac{U h_{0}}{2 a}\left[\cos m z-\frac{\left(1-\epsilon^{2}\right) \sin 4 \pi \hat{H}}{2\left(\epsilon^{2} \cos ^{2} 2 \pi \hat{H}+\sin ^{2} 2 \pi \hat{H}\right)} \sin m z\right]$,

which matches the expression for $w\left(x_{u}, z\right)$ for the single Fourier component (11) to within a constant factor.

To estimate the precipitation rate under saturated conditions, let us assume, following SB04, that the condensation rate $S$ can be approximated as 


$$
S(x, z) \approx S_{0} e^{-z / H_{w}} w(x, z),
$$

where $H_{w}$ is the moisture-scale height and $S_{0}$ is the condensation per unit vertical displacement at the surface. Let us further assume-very crudely-that total precipitation at the surface is equal to the vertical integral of condensation upstream of the crest:

$$
P_{\mathrm{est}}=\int_{0}^{\infty} \int_{-\infty}^{0} S(x, z) d x d z
$$

In nature, moisture tends to be concentrated in the lower troposphere, implying that

$$
e^{-H / H_{w}} \ll 1 .
$$

As a result, the stratospheric component of $w$ can be neglected in (33). Combining (28), (33), and (34), we arrive at the following estimate for precipitation:

$P_{\mathrm{est}}=\frac{S_{0} H_{w} U h_{0}}{1+\hat{H}_{w}^{2}}\left[1-\hat{H}_{w} \frac{\left(1-\epsilon^{2}\right) \sin 4 \pi \hat{H}}{2\left(\epsilon^{2} \cos ^{2} 2 \pi \hat{H}+\sin ^{2} 2 \pi \hat{H}\right)}\right]$,

where $\hat{H}_{w}=H_{w} N^{\prime} / U$ is a nondimensional moisturescale height, which determines the extent to which condensation depends on mountain-wave dynamics. ${ }^{3}$

To understand the full implications of (36), it is helpful first to consider the limit $\epsilon \rightarrow 1$ in which the tropopause vanishes. In this case, the quantity within parentheses approaches unity, and (36) simplifies to

$$
P_{\mathrm{est}}=\frac{S_{0} H_{w} U h_{0}}{1+\hat{H}_{w}^{2}} .
$$

Since we are specifically interested in the relationship between mountain-wave dynamics and precipitation, we assume that the thermodynamic quantities $S_{0}$ and $H_{w}$ are constant and focus instead on two ways in which $U$ and $N^{\prime}$ influence (37).

First, $U$ and $N^{\prime}$ together determine the vertical wavelength $\lambda_{z}$, which is inversely proportional to $\hat{H}_{w}$ in the denominator of (37). An increase in $U$, or a decrease in $N^{\prime}$, will cause $\hat{H}_{w}$ to decrease, thereby enhancing precipitation. Physically, this change in $P_{\text {est }}$ results from an increase in the depth of windward ascent, which in the absence of a tropopause is proportional to $\lambda_{z}$.

\footnotetext{
${ }^{3}$ When $\hat{H}_{w} \ll 1, P_{\text {est }}$ depends little on the vertical structure of the mountain waves, since moisture is confined near the surface where $w$ is fixed by the lower boundary condition (5). As $\hat{H}_{w}$ increases, however, mountain waves become more important as higher altitudes contribute a larger fraction of total condensation, incorporating more of the wave structure into the solution.
}

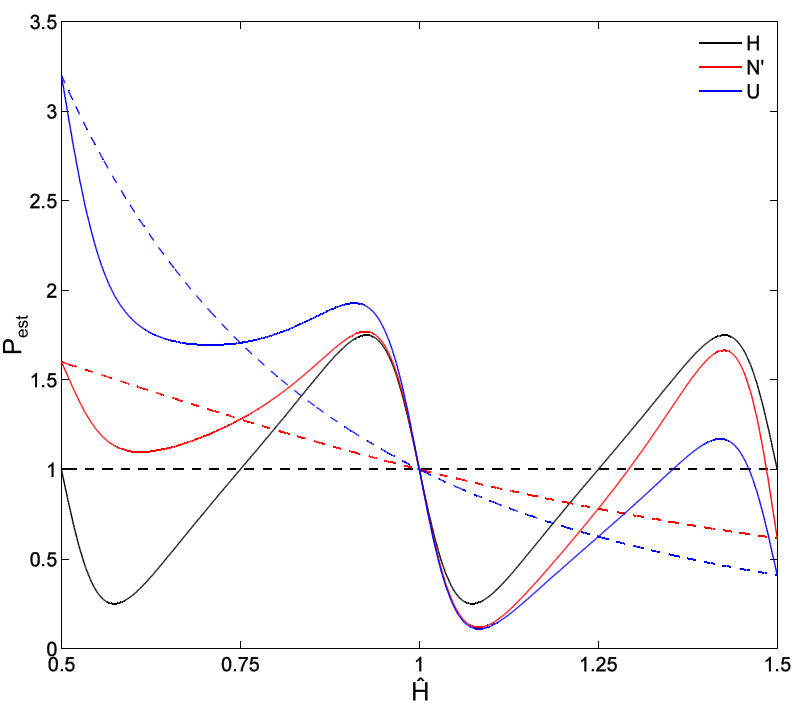

FIG. 2. The quantity $P_{\text {est }}$ as a function of $\hat{H}$, but with three different variables $\left(H, N^{\prime}\right.$, or $\left.U\right)$ acting as the independent variable in (36). To facilitate comparison, each curve has been normalized to 1 at $\hat{H}=1$. Black curves: variable $H$, fixed $U$ and $N^{\prime}$. Red curves: variable $N^{\prime}$, fixed $U$ and $H$. Blue curves: variable $U$, fixed $N^{\prime}$ and $H$. Solid lines represent a two-layer atmosphere with $\epsilon=0.5$, while dashed lines represent the limit $\epsilon \rightarrow 1$ [see (37)]. All curves use $\hat{H}_{w}=1$ for the nondimensional moisture-scale height.

Second, in addition to influencing the depth of ascent via $\lambda_{z}, U$ also controls the magnitude of ascent via the lower boundary condition in (5), as indicated in the numerator of (37). In a one-layer atmosphere, these two effects reinforce each other: an increase in $U$ enhances the magnitude as well as the depth of windward ascent, while a decrease in $U$ does the opposite. On the other hand, a change in $N^{\prime}$ only alters the depth of ascent, and will therefore have a smaller impact on $P_{\text {est }}$ than a change in $U$ of the same magnitude.

Returning to the more general expression for precipitation in (36), we find that, like low-level windward ascent, $P_{\text {est }}$ is diminished by wave reflection at the tropopause when $n<2 \hat{H}<n+(1 / 2)$ and is enhanced when $n-(1 / 2)<2 \hat{H}<n$. Furthermore, because $\hat{H}$ depends on $U$ and $N^{\prime}$, the actual relationship between $U, N^{\prime}$, and $P_{\text {est }}$ may be quite different than expected from the onelayer approximation in (37). The influence of these factors is illustrated in Fig. 2, where each curve plots $P_{\text {est }}$ as a function of $\hat{H}$, but with different variables $\left(H, N^{\prime}\right.$, or $U$ ) serving as the independent variable in (36). To facilitate comparison, each curve has been normalized to one at $\hat{H}=1$. In dimensional terms, it may be helpful to think of $\hat{H}=1$ as representing $H=12 \mathrm{~km}, U=15 \mathrm{~m} \mathrm{~s}^{-1}$, and $N^{\prime}=0.008 \mathrm{~s}^{-1}$. The black lines show the dependence of $P_{\text {est }}$ on $H$ assuming fixed $U$ and $N^{\prime}$. Since $H$ is proportional to $\hat{H}$, the $x$ axis spans values of $H$ ranging 
from $6 \mathrm{~km}$ at $\hat{H}=1 / 2$ to $18 \mathrm{~km}$ at $\hat{H}=3 / 2$. For the red lines, $U$ and $H$ are fixed while $N^{\prime}$ ranges from 0.004 to $0.012 \mathrm{~s}^{-1}$. For the blue lines, $N^{\prime}$ and $H$ are fixed while $U$ ranges from $30 \mathrm{~m} \mathrm{~s}^{-1}$ at $\hat{H}=1 / 2$ to $10 \mathrm{~m} \mathrm{~s}^{-1}$ at $\hat{H}=3 / 2$, reflecting the inverse relationship between $U$ and $\hat{H}$. Dashed lines represent the one-layer solution [see (37)] and serve both to highlight the effect of the tropopause and to illustrate the general slope of each curve.

When $H$ is the independent variable (black curve), $P_{\text {est }}$ is periodic with zero overall slope, indicating that changes in the height of the tropopause only affect the phase of the reflected mountain waves, not their vertical wavelength. In contrast, when $N^{\prime}$ is the independent variable (red curve), $P_{\text {est }}$ exhibits a negative slope on top of periodic fluctuations as $N^{\prime}$ increases from left to right. This behavior is due to the fact that $N^{\prime}$ influences both the wavelength and the phase of the reflected waves. The negative slope is even greater in the case where $U$ is the independent variable (blue curve), as a result of weaker ascent at the lower boundary as $U$ decreases from left to right. Yet even with the blue curve, the general tendency for $P_{\text {est }}$ to vary inversely with $\hat{H}$ is sometimes overcome as the effects of wave reflection at the tropopause generate local regions with positive slope. Such instances of positive slope suggest that decreases in $U$ (blue curve) or increases in $N^{\prime}$ (red curve) can sometimes increase orographic precipitation. This surprising result will be explored further in the next section.

The direct impact of the tropopause on orographic precipitation is evident in the difference between the dashed and solid lines in Fig. 2. Over the range of typical atmospheric conditions the one- and two-layer approximations can differ by more than a factor of 2 , suggesting that wave reflection by the tropopause should be considered among the most important factors controlling orographic precipitation. Still, it is important to keep the preceding analysis in perspective. Though concise, (36) is without question a very crude approximation of orographic precipitation in nature. In addition to linear dynamics, (36) is based on extremely simple thermodynamics [see (33)] and ignores cloud microphysics altogether. To better estimate the impact of the tropopause on orographic precipitation, we must resort to more sophisticated numerical simulations.

\section{Numerical simulations}

In this section, we take a step closer to reality, employing a nonlinear, nonhydrostatic mesoscale model to examine the influence of the tropopause on orographic precipitation in a series of idealized two- and threedimensional simulations. The model is an updated version of that fully described in Durran and Klemp (1983).
The model uses terrain-following coordinates and a two-time-step partially split approximation to the full compressible equations, updated to use third-order Runge-Kutta time differencing for the large-time-step integrations (Wicker and Skamarock 2002). The subgridscale turbulence formulation is based on Lilly (1962), and warm-rain microphysics are included through a Kessler (1969) parameterization. Ice microphysics are neglected for simplicity.

All simulations were performed with a resolution of $\Delta x=\Delta y=1 \mathrm{~km}$ in the horizontal and $\Delta z=200 \mathrm{~m}$ in the vertical. For the $2 \mathrm{D}$ simulations we used $800 \times 75 x-z$ grid points, while the grid for the 3D simulations contains $600 \times 600 \times 75$ grid points. Simulations were initialized from a state of rest and integrated forward with a large time step of $10 \mathrm{~s}$ until an approximate steady state was reached $(15 \mathrm{~h})$. To minimize the spurious artifacts associated with a cold start, the upstream wind speed was gradually increased from zero to its steady-state value over the first $2 \mathrm{~h}$ of each simulation. Open (radiation) boundary conditions were approximated at the lateral boundaries by propagating perturbations in the velocity component normal to the boundary outward at a phase speed of $25 \mathrm{~m} \mathrm{~s}^{-1}$. An open boundary condition was approximated at the top of the domain using a nonperiodic formulation of the Klemp-Durran-Bougeault hydrostatic gravity wave radiation condition that perfectly transmits wavelengths of 15, 60, and $120 \mathrm{~km}$ (Durran 2010, p. 484; Bougeault 1983; Klemp and Durran 1983).

We present three sets of experiments designed to test different aspects of the linear theory presented above. In the first set of experiments, we simulate dry flow over a low ridge to demonstrate the ability of the numerical model to reproduce the analytic flow-field solution under approximately linear conditions. In the second set of simulations, we raise the height of the ridge and evaluate the sensitivity of precipitation to variations in $H, U$, and $N$ under saturated conditions, comparing the results with (36). Finally, we present results of more realistic three-dimensional simulations involving a ridge of finite length and linear wind shear.

\section{a. 100-m ridge, dry troposphere}

We begin with simulations of dry flow over a low twodimensional witch-of-Agnesi ridge with dimensions $h_{0}=$ $100 \mathrm{~m}$ and $a=25 \mathrm{~km}$. To isolate the impact of wave reflection at the tropopause, we hold $N$ and $U$ constant while varying $H$. For the upstream sounding, we set $N=$ $0.01 \mathrm{~s}^{-1}, N_{s}=0.02 \mathrm{~s}^{-1}$, and $U=15.92 \mathrm{~m} \mathrm{~s}^{-1}$, which implies a hydrostatic vertical wavelength in the troposphere of $\lambda_{z}=10 \mathrm{~km}$. Simulations are performed with tropopause heights of $8.5,9.5,10.5$, and $11.5 \mathrm{~km}$, corresponding 

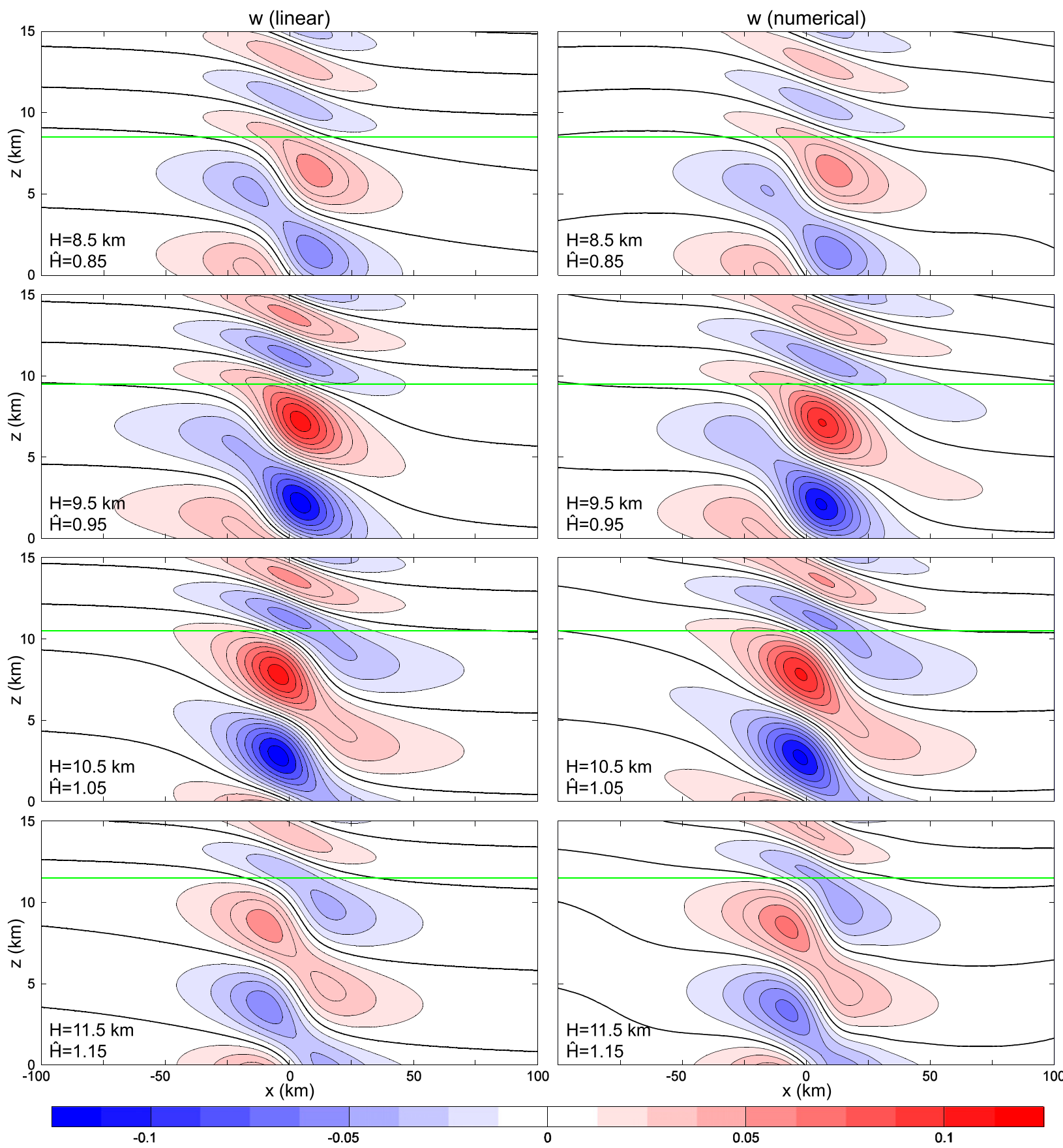

0.1

FIG. 3. Vertical velocities $\left(\mathrm{m} \mathrm{s}^{-1}\right)$ over a 100-m-high witch-of-Agnesi ridge in a two-layer atmosphere, (left) derived from linear theory [see (28)] and (right) a numerical model. Red (blue) colors indicate regions of ascent (descent), with thick black lines representing the zero contour. Green lines represent the tropopause. Solutions are shown for tropopause heights: (top to bottom) $H=8.5,9.5,10.5$, and $11.5 \mathrm{~km}$ and $\hat{H}=0.85,0.95,1.05$, and 1.15 .

to nondimensional tropopause heights of $\hat{H}=0.85,0.95$, 1.05, and 1.15 .

Figure 3 shows the vertical velocity fields for each simulation (right column), along with equivalent fields predicted by linear theory [see (28)] (left column). To compare the linear and numerical solutions directly, it is necessary to account for our use of the Boussinesq approximation in deriving the linear solution. To do so, we have scaled the fields from the numerical model by a factor of $\left(\rho / \rho_{0}\right)^{1 / 2}$, where $\rho_{0}$ is the air density at the surface and $\rho(z)$ is the 
density of the background flow as a function of height. Although this scaling is only exact as a transformation between Boussinesq and isothermal atmospheres, it is sufficiently accurate for our purposes, as demonstrated by the similarity in the magnitude of vertical velocities within the linear and numerical solutions in Fig. 3.

Significant changes to the flow field occur as $\hat{H}$ varies between 0.85 and 1.15. Upstream of the ridge crest (i.e., $x<0$ ), low-level ascent is deeper and more vigorous when $\hat{H}<1$ (i.e., when $H=8.5$ and $9.5 \mathrm{~km}$ ) than when $\hat{H}>1$ (i.e., $H=10.5$ and $11.5 \mathrm{~km}$ ), which is consistent with our analysis of windward ascent discussed in the previous section (Fig. 1). Other differences are also apparent, most notably in the magnitude and location of maximum leeside descent. Such differences will turn out to be important in later simulations, when we compare the distributions of precipitation across the ridge.

Overall, the numerical model does a good job of capturing variability in ascent associated with changes in tropopause height. The strong agreement between the linear and numerical fields in Fig. 3 demonstrates both the ability of the model to simulate wave reflection at the tropopause and the diversity of flow-field patterns that can result from it.

\section{b. 1-km ridge, saturated troposphere}

Our next series of simulations involves a twodimensional ridge of the same $25-\mathrm{km}$ half-width as before, but with a height of $1 \mathrm{~km}$ capable of generating significant precipitation. We consider separately three scenarios, in which one of the variables $H, N$, or $U$ is varied while the other two variables are held fixed. All simulations were performed using a surface temperature of $5^{\circ} \mathrm{C}$ and relative humidities of $100 \%$ in the troposphere and $20 \%$ in the stratosphere.

\section{1) THE RESPONSE OF PRECIPITATION TO CHANGING TROPOPAUSE HEIGHT}

Let us first consider how changes in $H$ affect precipitation while $U$ and $N$ are held constant. Thirteen simulations were performed with tropopause heights between 7.5 and $13.5 \mathrm{~km}$, each with upstream soundings of $U=15 \mathrm{~m} \mathrm{~s}^{-1}, N=0.012 \mathrm{~s}^{-1}$, and $N_{s}=0.02 \mathrm{~s}^{-1}$. From (24), we find that these conditions imply a moist stability, $N_{m}$, ranging from about $0.005 \mathrm{~s}^{-1}$ at the surface to $0.012 \mathrm{~s}^{-1}$ above $10 \mathrm{~km}$.

The blue dots in Fig. 4 show the cross-mountainintegrated precipitation rate for each simulation. The shape of the dots is approximately periodic, as predicted by linear theory [see (36)]. The effective stability, $N^{\prime}$, can be estimated from the phase of the dots, which suggests that $\hat{H}=1$ near $H=10.5 \mathrm{~km}$, with more (less) precipitation for tropopause heights below (above) that

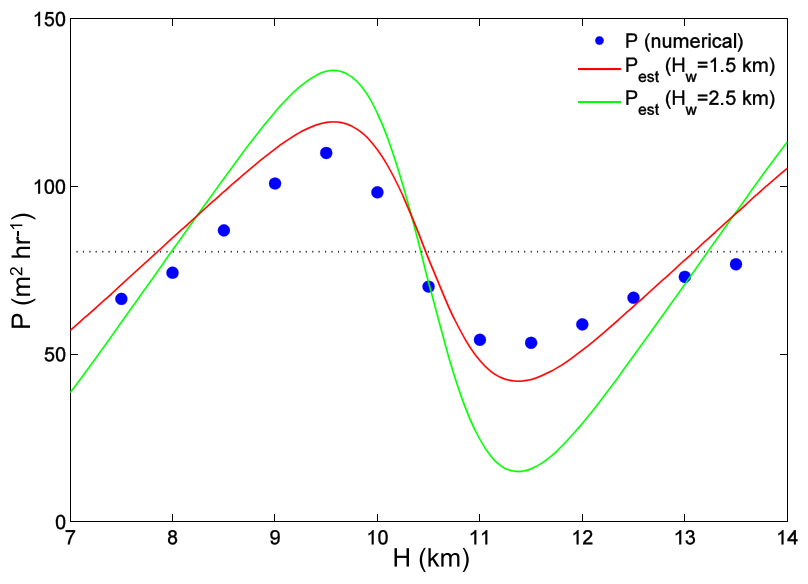

FIG. 4. Cross-ridge-integrated precipitation rates produced by two-dimensional saturated flow over a 1-km-high ridge as a function of tropopause height $H$ when $\epsilon=0.6$. Results are from nonlinear numerical simulations (blue dots) and the linear estimate [see (36)] with $H_{w}=1.5$ (red line) or $2.5 \mathrm{~km}$ (green line). For reference, the precipitation in the one-layer limit, $\epsilon=1$, (37) is also shown (dotted black line).

level. Since $U=15 \mathrm{~m} \mathrm{~s}^{-1}$, this implies an effective static stability of $N^{\prime} \approx 0.009 \mathrm{~s}^{-1}$, which is very close to the average of $\bar{N}_{m}$ across all simulations $\left(0.0091 \mathrm{~s}^{-1}\right)$. Significantly, this result appears to validate our earlier prediction-based on Jiang (2003) — that $N^{\prime} \approx \bar{N}_{m}$ when there are widespread regions of saturation and $N_{m}$ is significantly greater than zero. Overall, precipitation varies by more than a factor of two across the simulations (53$\left.110 \mathrm{~m}^{2} \mathrm{~h}^{-1}\right)$, with the greatest precipitation occurring when $H=9.5 \mathrm{~km}$ and the least when $H=11.5 \mathrm{~km}$.

How do these results compare to the linear approximation [see (36)] derived in the previous section? Given $S_{0}=1.9 \times 10^{-3} \mathrm{~g} \mathrm{~m}^{-4}$ (see appendix), an effective stability of $N^{\prime}=0.009 \mathrm{~s}^{-1}$, and a moisture-scale height of $H_{w}=1.5 \mathrm{~km}$, the linear approximation agrees well with the numerical simulations (red line). However, for the conditions simulated $\left(T_{s}=5^{\circ} \mathrm{C}, N=0.012 \mathrm{~s}^{-1}\right)$, the actual $e$-folding height of water vapor is around $2.5 \mathrm{~km}$ (see appendix). Using this more realistic value for $H_{w},(36)$ significantly overpredicts the sensitivity of precipitation to changes in tropopause height (green line).

There are at least two possible reasons why using a lower value of $H_{w}$ in (36) produces better agreement with the numerical simulations, both of which are evident in Fig. 5, which shows the linear and numerical vertical velocity fields for the cases of maximum and minimum precipitation $(H=9.5$ and $11.5 \mathrm{~km})$. First, (36) is based on the assumption that precipitation is equal to upstream condensation, which neglects the microphysical processes and time scales involved in converting condensation into precipitation. In reality, 

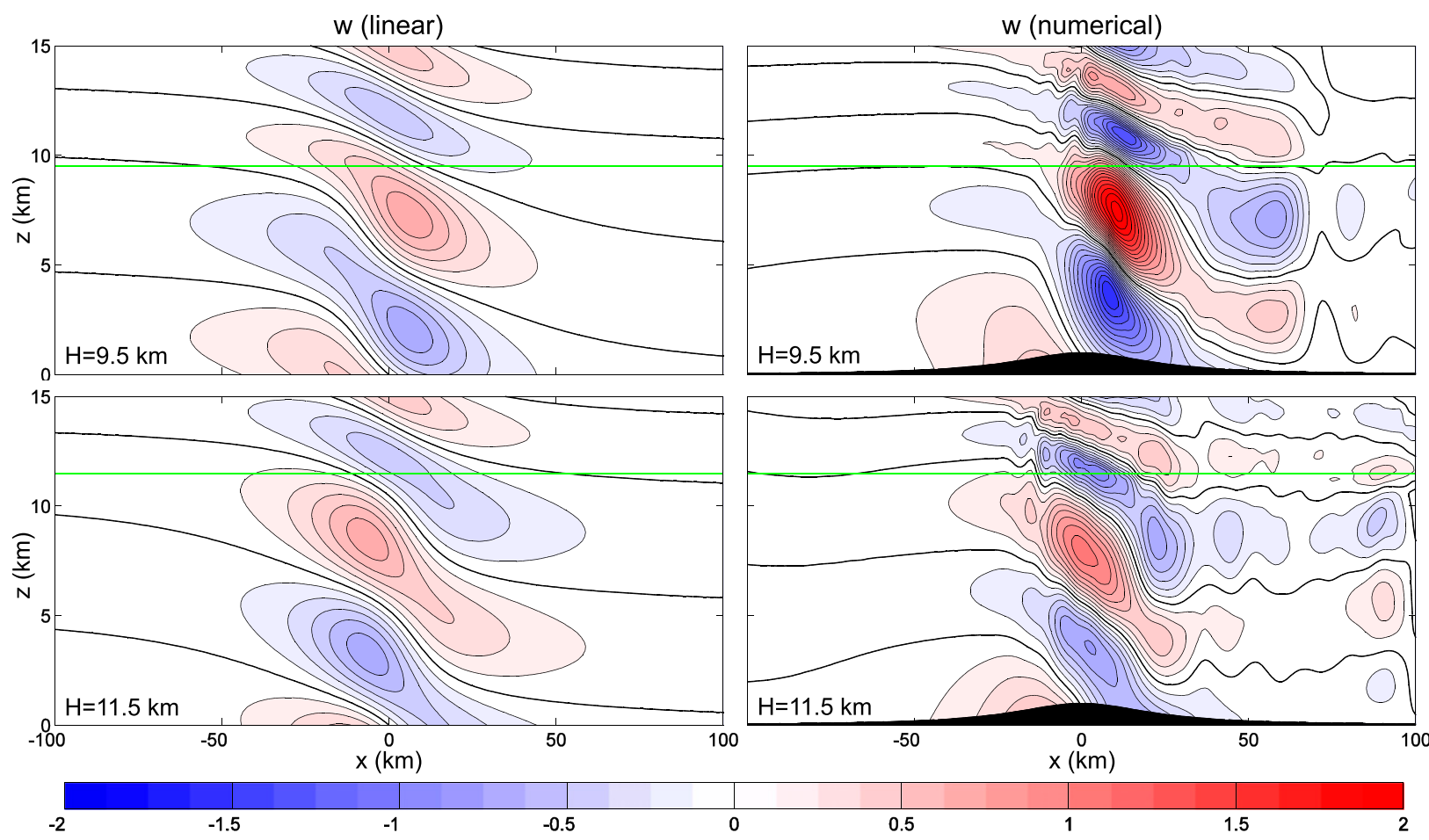

FIG. 5. As in Fig. 3, except for a 1-km-high ridge; and solutions are shown for the two numerical cases: (top) $H=9.5$ and (bottom) $11.5 \mathrm{~km}$, exhibiting the most and least precipitation, respectively. Units are $\mathrm{m} \mathrm{s}^{-1}$.

some of the condensation that forms aloft evaporates in the lee before reaching the surface, especially when windward ascent is deepest (e.g., when $H=9.5 \mathrm{~km}$; topleft panel). Second, (33) [from which (36) is derived] is indifferent to the sign of $w$, treating regions of descent as negative sources of condensation (i.e., evaporation). Therefore, when windward ascent is shallow and topped by a layer of vigorous descent (as in the $11.5-\mathrm{km}$ case; bottom-left panel), the linear column-integrated condensation is negative over portions of the windward slope near the crest. In such cases, (36) gives too much weight to evaporation aloft at the expense of condensation near the surface. Both of these problems are mitigated by using a lower moisture-scale height, likely explaining the improved agreement between the linear and numerical results.

To better understand the connection between vertical velocity and precipitation within the numerical simulations, it is useful to compare the $H=9.5$ - and $11.5-\mathrm{km}$ cases in greater detail. Figures $6 \mathrm{a}$ and $6 \mathrm{~b}$ show the concentration of cloud water (blue lines) and rainwater (shading), in addition to streamlines of parcels originating $100 \mathrm{~km}$ upstream of the ridge crest (red lines). Focusing first on the streamlines, we find that for a parcel that begins at $1 \mathrm{~km}$ altitude, the differences between the two cases are modest, with only about $150 \mathrm{~m}$ more ascent in the $9.5-\mathrm{km}$ case. However, for a parcel originating at 3 or $4 \mathrm{~km}$, where the flow is less influenced by the free-slip lower boundary condition, the difference in maximum ascent between the two cases is much larger $(\sim 400 \mathrm{~m})$. In the $11.5-\mathrm{km}$ case, in fact, the $4-\mathrm{km}$ streamline actually descends over the windward slope, falling more than $150 \mathrm{~m}$ by the time it crosses the ridge crest. This explains why the cloud layer is both deeper and thicker in the $9.5-\mathrm{km}$ case, resulting in more than twice as much precipitation.

A further contrast between the two cases is evident downwind of the crest, where each streamline in the 9.5-km case descends lower than its counterpart in the $11.5-\mathrm{km}$ case. This behavior is caused by much stronger leeside descent in the $9.5-\mathrm{km}$ case (Fig. 5), and it has important consequences for leeside evaporation: in the $9.5-\mathrm{km}$ case, no cloud water is present beyond $15 \mathrm{~km}$ downwind of the crest, while in the $11.5-\mathrm{km}$ case, cloud water persists more than $30 \mathrm{~km}$ downwind of the crest owing to weaker descent and lower evaporation rates.

These differences in leeside descent-evaporation also affect precipitation, as shown in Fig. 6c. While the 9.5-km case exhibits greater precipitation overall, the ratio of leeward-to-windward precipitation is significantly lower in the $9.5-\mathrm{km}$ case than in the $11.5-\mathrm{km}$ case (0.35 versus 0.51 ), indicative of a stronger orographic 

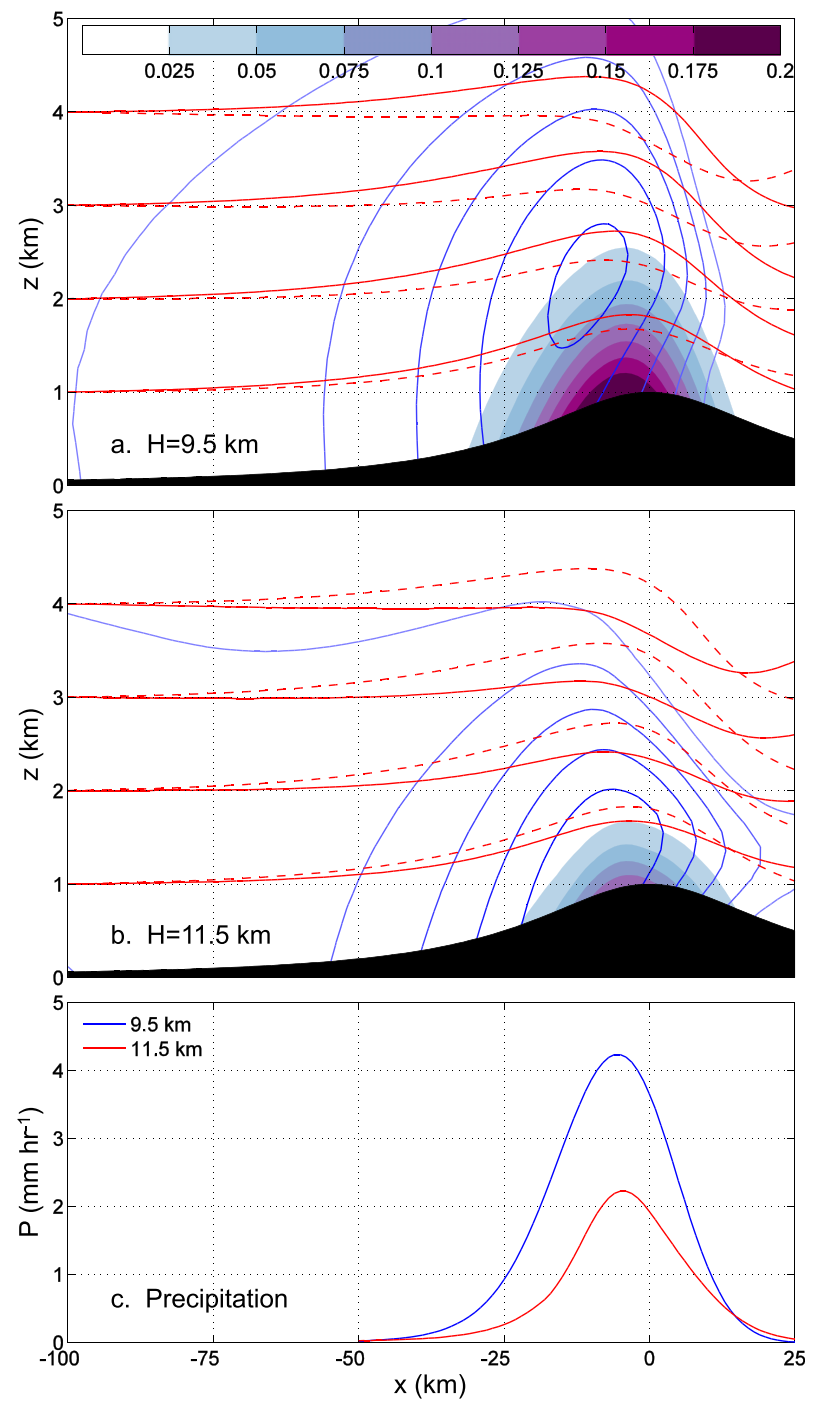

FIG. 6. Cloud water mixing ratio (blue contours, intervals of $0.2 \mathrm{~g} \mathrm{~kg}^{-1}$ ), rainwater mixing ratio (blue shading, $\mathrm{g} \mathrm{kg}^{-1}$ ), and parcel streamlines (red lines) from numerical simulations in which $H$ is (a) 9.5 and (b) $11.5 \mathrm{~km}$. Dashed red lines show the streamlines from the other simulation. (c) Precipitation rates as a function of the cross-ridge coordinate for both cases $\left(\mathrm{mm} \mathrm{h}^{-1}\right)$.

rain shadow. In section 4 , we discuss the possible implications of this result for rain-shadow variability in realistic terrain.

\section{2) THE RESPONSE OF PRECIPITATION TO CHANGING WIND SPEED AND STATIC STABILITY}

In addition to varying $H$, further simulations were performed varying $U$ (from 10 to $20 \mathrm{~m} \mathrm{~s}^{-1}$, with $N=$ $0.012 \mathrm{~s}^{-1}$ ) and $N$ (from 0.009 to $0.015 \mathrm{~s}^{-1}$, with $U=$ $15 \mathrm{~m} \mathrm{~s}^{-1}$ ). All other parameters where held constant, including $N_{s}=0.02 \mathrm{~s}^{-1}, H=10.5 \mathrm{~km}$, and the surface temperature at $5^{\circ} \mathrm{C}$.
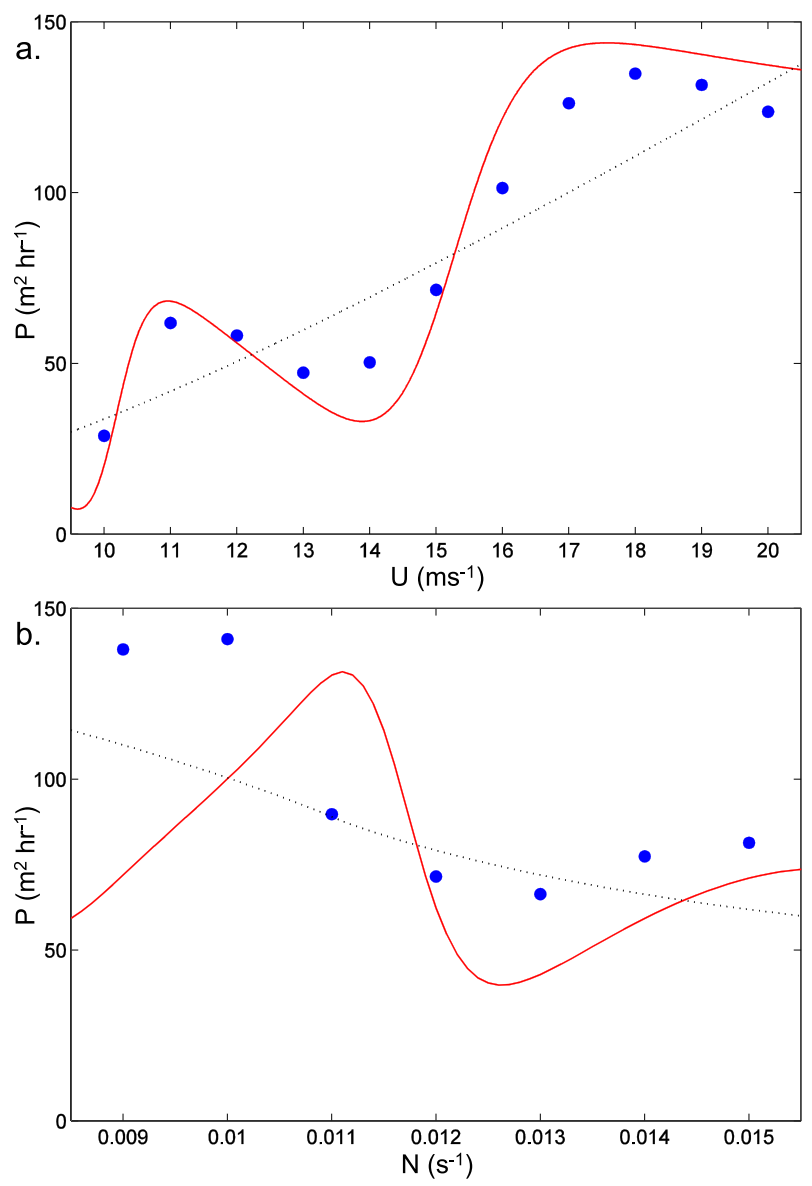

FIG. 7. Cross-ridge-integrated precipitation rates produced by saturated flow over a 1-km-high ridge as a function of (a) wind speed and (b) static stability, from numerical simulations (blue dots) and (36) (red lines), calculated assuming $N^{\prime}=\bar{N}_{m}$.

Figure 7a shows the cross-mountain-integrated precipitation rate from the variable- $U$ simulations (blue dots), alongside the linear approximation [see (36)] (red line), calculated using $S_{0}=1.9 \times 10^{-3} \mathrm{~g} \mathrm{~m}^{-4}, H_{w}=$ $1.5 \mathrm{~km}$, and $N^{\prime}=\bar{N}_{m}=0.009 \mathrm{~s}^{-1}$. Overall, the linear approximation does a good job of capturing both the amplitude and phase of precipitation variability-further evidence that $N^{\prime} \approx \bar{N}_{m}$ is accurate under these conditions. While faster wind speeds generally result in greater precipitation, this tendency is reversed over certain intervals of $U$ (between 11 and 13 and 18 and $20 \mathrm{~m} \mathrm{~s}^{-1}$ ), just as predicted in the linear approximation. That such behavior would be impossible in a one-layer atmosphere (dotted line) demonstrates the significant impact the tropopause can have on orographic precipitation.

Calculating $P_{\text {est }}$ is trickier when $N$ is the independent variable (Fig. 7b), because $N^{\prime}$ can no longer be identified from the phase of the numerical results (blue dots). If we assume, based on previous results, that $N^{\prime}=\bar{N}_{m}$, the 
linear approximation (red line) performs reasonably well when $N \geq 0.012 \mathrm{~s}^{-1}$ but rather poorly when $N<$ $0.012 \mathrm{~s}^{-1}$, suggesting that linear theory breaks down as $N$ decreases and the lower troposphere approaches convective instability. Overall, the influence of the tropopause is clear in both the linear and numerical results, which show precipitation increasing as $N$ is raised from 0.013 and $0.015 \mathrm{~s}^{-1}$ - a result that would not be expected in the absence of a tropopause (dotted line).

Together, Figs. 4 and 7 demonstrate two things. First, despite its simplicity, (36) is nevertheless a useful tool for understanding the connection between windward ascent and orographic precipitation. Second, within a nonlinear, nonhydrostatic numerical model, the amount and distribution of orographic precipitation is significantly affected by wave reflection at the tropopause, just as one would expect based on linear theory.

\section{c. $3 D$ ridge, linear shear}

Our third set of simulations incorporates two changes intended to better approximate nature: three-dimensional terrain and linear wind shear. We focus on a simple forward-shear scenario, in which $U$ increases linearly with height in the troposphere and is constant in the stratosphere. The linear flow field under these conditions has been discussed elsewhere (e.g., Klemp and Lilly 1975) and will not be repeated here. For our purposes, it is sufficient to note that the presence of wind shear does not change the essential result from section 2-that low-level windward ascent will either be enhanced or diminished depending on the phase of the mountain waves reflected by the tropopause. Using the same notation as before, and following the same procedure described by Klemp and Lilly (1975), it can be shown that for a tropospheric wind profile given by

$$
U(z)=U_{0}(1+\alpha z)
$$

the hydrostatic nondimensional tropopause height takes the form

$$
\hat{H}=\frac{1}{2 \pi \alpha}\left(\frac{N^{\prime 2}}{U^{2}}-\frac{\alpha^{2}}{4}\right)^{1 / 2} \ln (1+\alpha H) .
$$

While this equation is considerably more complicated than its no-shear equivalent [see (30)], $\hat{H}$ plays essentially the same role in both cases, indicating diminished lowlevel windward ascent when $n<2 \hat{H}<n+(1 / 2)$ and enhanced ascent when $n-(1 / 2)<2 \hat{H}<n$. Therefore, whether $U$ is constant or sheared, we expect precipitation to be similarly sensitive to variations in tropopause height.

To test this hypothesis, we have performed a series of $3 \mathrm{D}$ numerical simulations with tropopause heights between 7.5 and $13.5 \mathrm{~km}$, but with constant upstream soundings of $U_{0}=15 \mathrm{~m} \mathrm{~s}^{-1}, \alpha=1.67 \times 10^{-4} \mathrm{~m}^{-1}, N=$ $0.012 \mathrm{~s}^{-1}, 100 \%$ tropospheric relative humidity, and a surface temperature of $5^{\circ} \mathrm{C}$. From (38), these parameters imply that $U$ increases $2.5 \mathrm{~m} \mathrm{~s}^{-1}$ per kilometer of altitude within the troposphere. In the stratosphere, $N_{s}=$ $0.02 \mathrm{~s}^{-1}$ and $U=U_{0}(1+\alpha H)$. The terrain consists of a finite ridge given by

$$
h(x, y)=\left\{\begin{array}{lll}
\frac{h_{0} a^{2}}{a^{2}+x^{2}}, & \text { if } & |y| \leq l \\
\frac{h_{0} a^{2}}{a^{2}+x^{2}+(|y|-l)^{2}}, & \text { if } & |y|>l
\end{array},\right.
$$

where $h_{0}=1 \mathrm{~km}$ and $a=25 \mathrm{~km}$ are the height and halfwidth of the ridge as before, while $2 l=300 \mathrm{~km}$ is the length of the ridge in the $y$ dimension. At the upstream boundary, the direction of the background flow is assumed to be perpendicular to the ridge axis at all levels. Simulations were performed on the $f$ plane using Coriolis parameters of 0 and $10^{-4} \mathrm{~s}^{-1}$. Since the Coriolis term did not change the results substantially, here we discuss only the $f=0$ case.

As expected, precipitation within these simulations is found to vary significantly with $H$, just as it did in previous 2D simulations with constant $U$. Overall, the greatest precipitation occurs when $H=8.5 \mathrm{~km}$ and the least when $H=13 \mathrm{~km}$. Figure 8 shows the precipitation rate in each of these simulations on top of topographic contours (black lines). Along the bisect shown in green, the integrated cross-ridge precipitation rate differs by a factor of 1.75 between the two simulations $\left(80.2 \mathrm{vs} 45.9 \mathrm{~m}^{2} \mathrm{~h}^{-1}\right)$.

The reason for this difference in precipitation is evident in Figs. 9a,b, which show the concentrations of cloud water (blue lines), rainwater (blue shading), and parcel streamlines (red lines) in the vertical plane intersecting the green line in Fig. 8 . When $H=8.5 \mathrm{~km}$, parcels ascend higher over the windward slope (red lines), leading to greater concentrations of liquid water and ultimately precipitation (Fig. 9c), just as we saw in the 2D simulations (Fig. 6). Leeside descent also differs between the two simulations, though rain-shadow strength is less affected because the contrast in descent is greatest beyond $25 \mathrm{~km}$ downwind of the crest, where there is little liquid water to evaporate.

In summary, whether in two or three dimensions, uniform or sheared flow, the simulations presented in this section have demonstrated that the tropopause has a first-order impact on the amount and distribution of orographic precipitation. In the next section, we consider the possible implications of this result for precipitation variability in realistic terrain. 

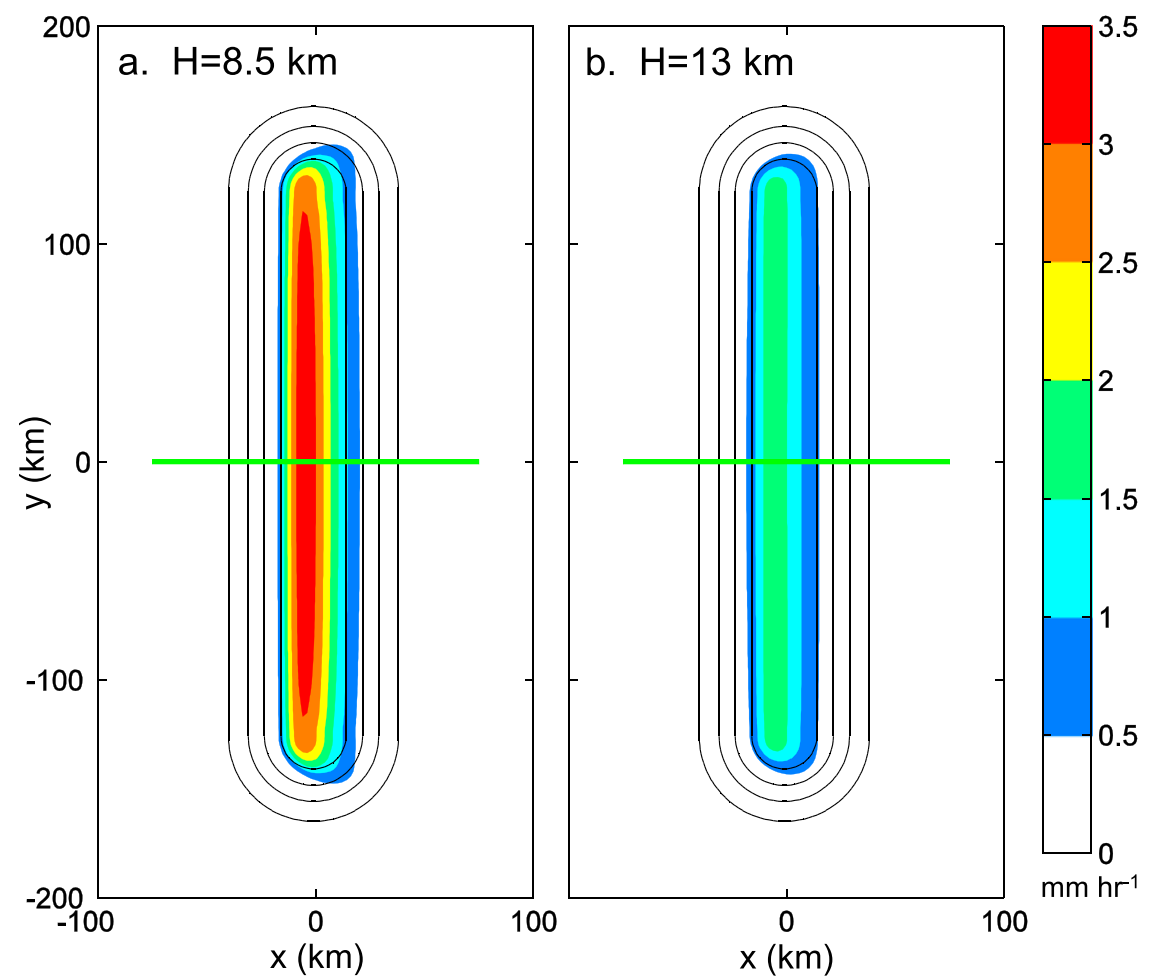

FIG. 8. Precipitation rate (shaded contours, $\mathrm{mm} \mathrm{h}^{-1}$ ) and terrain height (black contours, 200-m intervals) for the 3D simulations with (a) $H=8.5$ and (b) $H=13 \mathrm{~km}$. The flow is across the ridge from left to right. Along the green line bisecting the ridge, the cross-ridge-integrated precipitation rate is (a) 80.2 and (b) $45.9 \mathrm{~m}^{2} \mathrm{~h}^{-1}$.

\section{Application to realistic terrain using a modified version of the Smith-Barstad model}

In the previous section, we used a nonlinear numerical model to show that orographic precipitation is significantly influenced by the reflection of mountain waves at the tropopause, confirming our prediction based on linear theory. We also found that while the amount of precipitation seems to depend mostly on the depth and magnitude of windward ascent, the distribution of precipitation is also influenced by the magnitude of descent in the lee. In cases where windward ascent was most enhanced by the tropopause, leeside descent was also quite vigorous, resulting in a strong rain shadow. On the other hand, cases with the weakest windward ascent also exhibited weak leeside descent, resulting in less evaporation and a weaker rain shadow.

In light of these results, it is reasonable to ask what role the tropopause might play in controlling the amount and distribution of orographic precipitation in the real world. Here we attempt to shed some light on this question by using a modified version of the linear model of orographic precipitation of SB04 to compare rainfall patterns in the Washington Cascades given two different tropopause heights.

\section{a. Linear model description}

The SB04 model utilizes the same approximation for column-integrated condensation [see (33)] that we used to approximate precipitation over a 2D ridge [see (36)]. However, rather than integrating in space to calculate the total precipitation [see (34)], the SB04 model estimates the spatial distribution of precipitation using the following differential equations governing the columnintegrated densities of cloud water $q_{c}$ and rainwater $q_{r}$ :

$$
\begin{aligned}
& \mathbf{U} \cdot \nabla q_{c}=S(x, y)-q_{c} / \tau_{c} \quad \text { and } \\
& \mathbf{U} \cdot \nabla q_{r}=q_{c} / \tau_{c}-q_{r} / \tau_{f} .
\end{aligned}
$$

Here, $\mathbf{U}$ is the two-dimensional wind vector and $S(x, y)$ is the two-dimensional, column-integrated form of the condensation rate defined in (33). The constants $\tau_{c}$ and $\tau_{f}$ represent characteristic time scales for cloud water conversion and rainwater fallout and are, therefore, a simple way to incorporate cloud microphysics within 

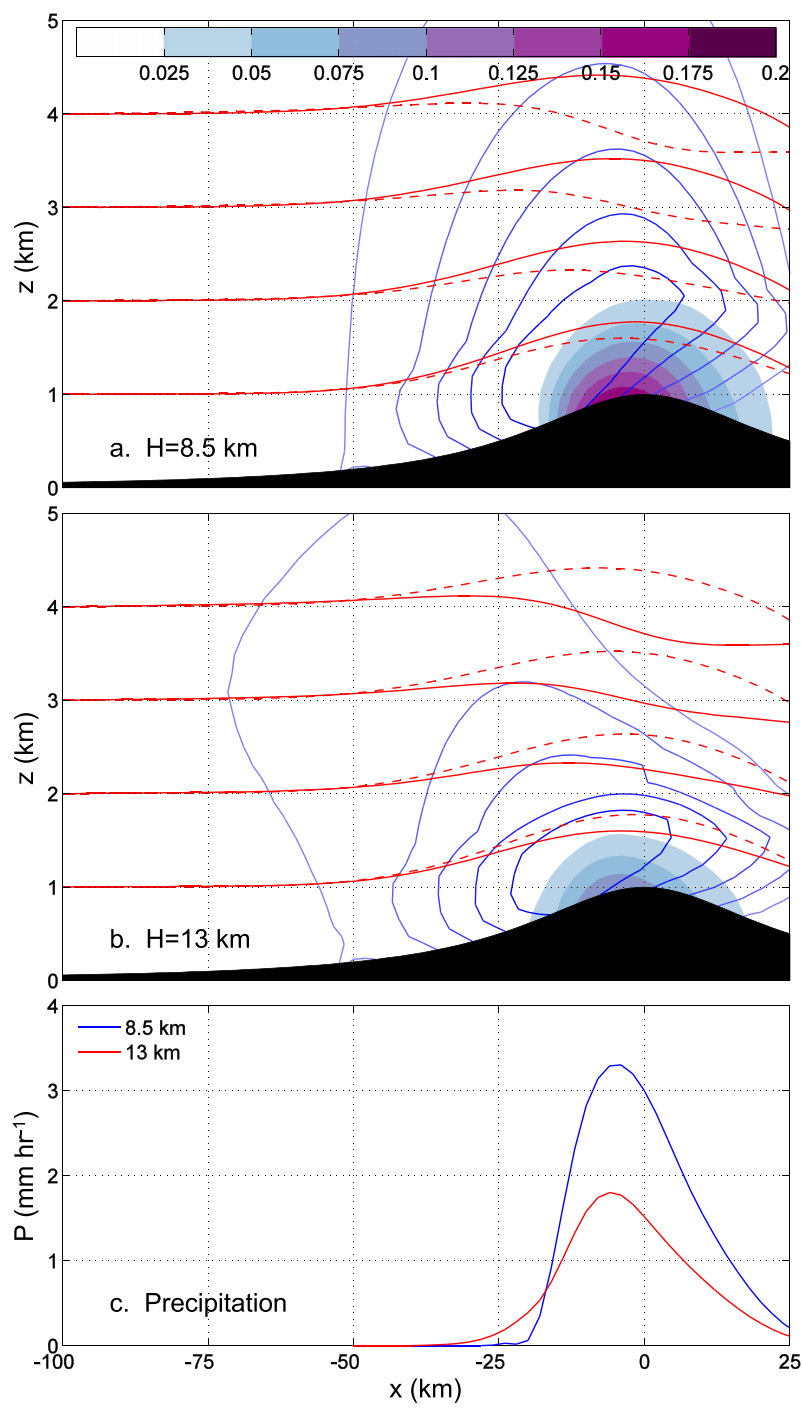

FIG. 9. Cloud water mixing ratio (blue contours, intervals of $0.2 \mathrm{~g} \mathrm{~kg}^{-1}$ ), rainwater mixing ratio (blue shading, $\mathrm{g} \mathrm{kg}^{-1}$ ), and parcel streamlines (red lines) along the bisecting plane of the ridge (green line, Fig. 8) in the 3D numerical simulations exhibiting the least and most precipitation: (a) $H=8.5$ and (b) $13 \mathrm{~km}$. Dashed red lines indicate streamlines from the other simulation. (c) Precipitation rates for the two cases $\left(\mathrm{mm} \mathrm{h}^{-1}\right)$.

a linear framework. In the second equation, the sink term $q_{r} / \tau_{f}$ is equivalent to the precipitation rate at the surface. By Fourier transforming these equations in the horizontal and rearranging the terms, the precipitation rate is found to be

$$
\hat{P}(k, l)=\frac{\hat{S}(k, l)}{\left(1+i \sigma \tau_{c}\right)\left(1+i \sigma \tau_{f}\right)},
$$

where $\hat{S}(k, l)$ represents the two-dimensional Fourier transform of the column-integrated condensation,

$$
\hat{S}(k, l)=S_{0} \int_{0}^{\infty} e^{-z / H_{w}} \hat{w}(k, l, z) d z,
$$

with $S_{0}$ and $H_{w}$ defined in (33). ${ }^{4}$

In the original SB04 model, $\hat{w}$ in (44) is calculated assuming a one-layer atmosphere with constant $N^{\prime}$ and $U$. An extension of the model, introduced by Barstad and Schüller (2011), accommodates multiple layers with different $N^{\prime}$ and $U$ and has been used to demonstrate how changes in wind speed and microphysical time scales affect precipitation. However, if we assume that (35) holds, and that $U, \tau_{c}$, and $\tau_{f}$ are constant everywhere, it is sufficient for our purposes to use the original model, but with $\hat{w}$ in (44) replaced by its two-layer analog within the troposphere. With this single modification, our model retains the simplicity of the original model, while still incorporating the effects of a tropopause.

Following the same procedure described in section 2 for a single Fourier component, the three-dimensional transformed tropospheric vertical velocity is found to be

$$
\hat{w}(k, l, z)=i \sigma \hat{h}(k, l)\left(C^{+} e^{i m z}+C^{-} e^{-i m z}\right),
$$

where the constants $C^{ \pm}$are defined in (8), $\hat{h}$ is the transformed height of the terrain, and $\sigma=U k+V l$, with $V$ and $l$ representing the wind speed and wavenumber in the $y$ dimension, analogous to $U$ and $k$ in the $x$ dimension. Given constant $N^{\prime}$ and $U$, the (nonhydrostatic) $2 \mathrm{D}$ vertical wavenumber is given by

$$
m^{2}=\frac{N^{\prime 2}-\sigma^{2}}{\sigma^{2}}\left(k^{2}+l^{2}\right) .
$$

Combining (43)-(45), we arrive at the full expression for the Fourier transformed precipitation rate,

$$
\hat{P}(k, l)=\frac{S_{0} H_{w} i \sigma \hat{h}(k, l)}{\left(1+i \sigma \tau_{c}\right)\left(1+i \sigma \tau_{f}\right)}\left(\frac{C^{+}}{1-i m H_{w}}+\frac{C^{-}}{1+i m H_{w}}\right),
$$

which is equivalent to (49) from SB04, but with the effects of a tropopause included. As in the original model, if we allow for a background precipitation rate, $P_{\infty}$, and exclude the possibility of negative precipitation, the precipitation rate in real space becomes

\footnotetext{
${ }^{4}$ Note that in the original version of the model, $S_{0}$ is replaced by $C_{w} / H_{w}$, and formulas are given for estimating both $C_{w}$ and $H_{w}$ from the surface temperature and lapse rate. However, it is our opinion that $S_{0}$ is more physically intuitive than $C_{w}$, since it represents the condensation per unit vertical displacement at the surface. An exact expression for $S_{0}$ was derived by Siler and Roe (2014) and is discussed in the appendix.
} 
a.

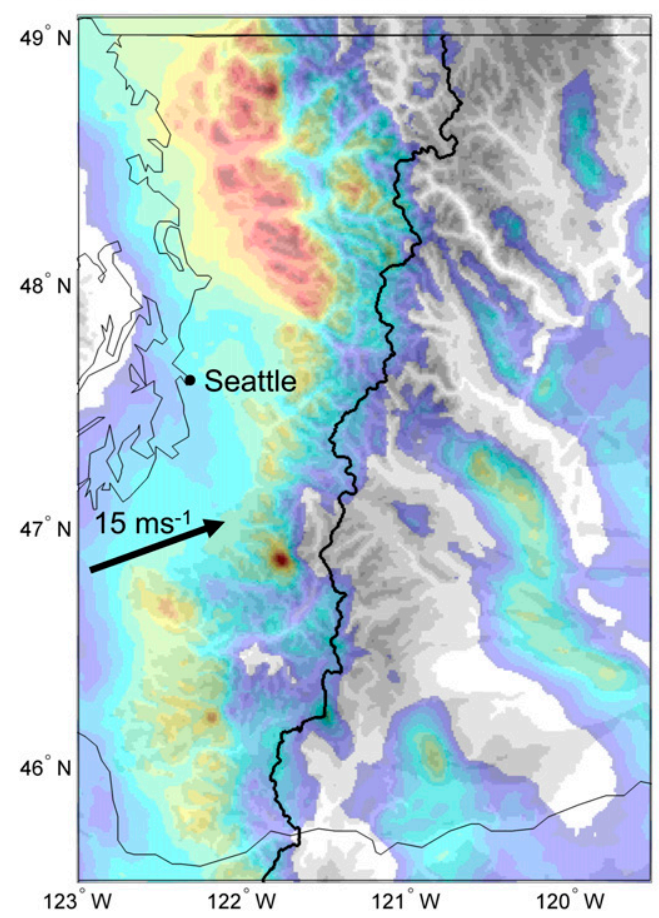

b. $\mathrm{H}=11.5 \mathrm{~km}$

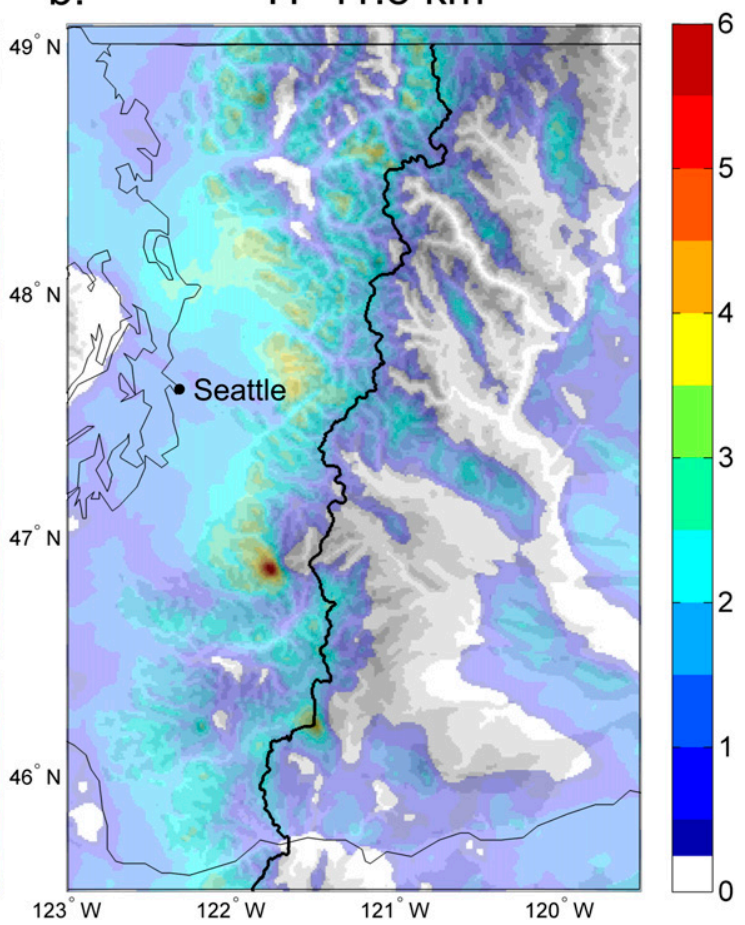

FIG. 10. The precipitation rate $\left(\mathrm{mm} \mathrm{h}^{-1}\right)$ in the Washington Cascades given tropopause heights of (a) 9.5 and (b) $11.5 \mathrm{~km}$, calculated using a modified version of the linear model of SB04. Input parameters are $N^{\prime}=0.009 \mathrm{~s}^{-1}$, $S_{0}=1.9 \times 10^{-3} \mathrm{~g} \mathrm{~m}^{-4}, H_{w}=2.5 \mathrm{~km},|\mathbf{U}|=15 \mathrm{~m} \mathrm{~s}^{-1}$ (from $250 \%$ west-southwest), $\tau_{c}=\tau_{f}=1000 \mathrm{~s}$, and $P_{\infty}=1 \mathrm{~mm} \mathrm{~h}^{-1}$. The black line represents the crest of the range.

$$
P(x, y)=\max \left(P^{*}, 0\right),
$$

where

$$
P^{*}(x, y)=\int_{-\infty}^{\infty} \int_{-\infty}^{\infty} \hat{P}(k, l) e^{i k x} e^{i l y} d k d l+P_{\infty} .
$$

\section{b. Application of the linear model to the Washington Cascades}

To illustrate the potential for tropopause height to influence precipitation patterns in realistic terrain, here we apply the modified linear model to the Cascades of Washington State, where significant variability in rainshadow strength has been observed (Siler et al. 2013). We present results for two different tropopause heights, with $\tau_{c}=\tau_{f}=1000 \mathrm{~s}$, and with other parameters unchanged from the 2D simulations presented in section 3 $\left(S_{0}=1.9 \times 10^{-3} \mathrm{~g} \mathrm{~m}^{-4}, N^{\prime}=0.009 \mathrm{~s}^{-1}\right)$. With microphysics now implicitly accounted for, the factors that necessitated a reduction in $H_{w}$ in section 3 no longer apply, and we therefore use the empirically derived value of $H_{w}=2.5 \mathrm{~km}$. Based on an analysis of Cascade storms by Siler et al. (2013), we set $P_{\infty}=1 \mathrm{~mm} \mathrm{~h}^{-1}$ and assume a wind speed of $15 \mathrm{~m} \mathrm{~s}^{-1}$ with a west-southwesterly orientation of $250^{\circ}$. Calculations were made using a
$1024 \times 1024$ grid with $1-\mathrm{km}$ resolution centered on the Washington Cascades (not shown).

Figure 10 shows the precipitation patterns in the Washington Cascades predicted by the linear model for tropopause heights of 9.5 and $11.5 \mathrm{~km}$. These patterns exhibit similar differences as the 2D numerical simulations (Fig. 6c). First, precipitation is significantly greater in the $9.5-\mathrm{km}$ case as a result of enhanced ascent over the windward slope. Second, the distribution of precipitation is more evenly distributed between eastern and western slopes in the $11.5-\mathrm{km}$ case than in the $9.5-\mathrm{km}$ case, with ratios of eastern-to-western precipitation equal to 0.42 and 0.30 , respectively. As in the $2 \mathrm{D}$ simulations, the contrast in precipitation patterns is a result of both weaker windward ascent and weaker leeside descent in the $11.5-\mathrm{km}$ case, which allows cloud water to penetrate further downstream. These results suggest that wave reflection by the tropopause may account for some of the variability in rain-shadow strength observed among storms in the Cascades (Siler et al. 2013), though more observations are needed to evaluate this theory with any confidence.

\section{Summary and discussion}

In this paper, we have used a combination of linear theory and numerical simulations to investigate the 
impact of the tropopause on orographic precipitation. Our main results are summarized below.

(i) According to linear theory, wave reflection at the tropopause can either enhance or diminish lowlevel windward ascent, depending on the value of a nondimensional number $\hat{H}$, which represents the ratio of the tropopause height to the vertical wavelength of the mountain waves. When the tropopause lies a bit below an integer multiple of a half vertical wavelength (i.e., when $n-(1 / 2)<2 \hat{H}<n$ ) low-level windward ascent is enhanced. Conversely, ascent is diminished when the tropopause lies a bit above an integer multiple of a half vertical wavelength [i.e., when $n<2 \hat{H}<n+(1 / 2)]$.

(ii) Combining linear dynamics with crude assumptions about condensation and hydrometeor fallout, we derived an approximation for precipitation over a $2 \mathrm{D}$ ridge $\left[P_{\text {est }}\right.$; see (36)], which exhibits the same sensitivity to $\hat{H}$ as windward ascent. This equation implies that the tropopause can exert just as much influence on orographic precipitation as wind speed and static stability, whose influence on precipitation is well documented.

(iii) Numerical simulations of saturated flow over a 2D ridge were performed with a range of wind speeds, static stabilities, and tropopause heights. In general, total precipitation within the simulations was found to agree well with the linear approximation when the effective static stability, $N^{\prime}$, was assumed to be equal to the troposphere-mean moist static stability, $\bar{N}_{m}$. The simulations also confirm one of the more surprising predictions of the linear approximationthat an increase in $U$ or a decrease in $N$ can in some cases result in less orographic precipitation.

(iv) Further simulations were performed with the same range of tropopause heights as before, but on a 3D grid with linear wind shear. These changes did not dramatically affect the sensitivity of precipitation to tropopause height, as precipitation varied by a factor of 2.06 in the 2D simulations and 1.75 in the 3D simulations.

(v) The idealized simulations showed significant differences not only in the amount of precipitation, but also in the strength of the orographic rain shadow. The contrast in rain-shadow strength is due to differences in the magnitude of leeside descent and, thus, evaporation. To test the possible implications of this result in realistic terrain, we introduced a version of the linear orographic precipitation model of SB04, which we modified to account for the presence of a tropopause. Using the Washington Cascades as a case study, we showed that modest changes in tropopause height do in fact have a significant impact on both the amount of precipitation and the strength of the rain shadow. This suggests that wave reflection by the tropopause could account for some of the variability in rain-shadow strength observed among major Cascade storms.

How important is the tropopause to orographic precipitation in nature? Observations and more sophisticated modeling studies may eventually help answer this question, but for now we can only speculate. Climatologically, we suspect that in most mountain ranges, $\hat{H}$ is sufficiently variable that the tropopause has little impact on mean precipitation patterns. For a given storm, however, the influence of the tropopause likely depends on the environmental conditions.

Two types of environments are particularly unfavorable to strong tropopause influence. First, when storms exhibit a combination of strong winds and low static stability-as might occur within an atmospheric river, for example-the vertical wavelength can greatly exceed the tropopause height, such that $\hat{H} \approx 0$. From (36), this implies that precipitation should scale linearly with wind speed, consistent with the high correlation between wind speed and precipitation observed during high-wind, low-stability storms in California's coastal mountains (Neiman et al. 2002). On the opposite end of the spectrum, when storms with weak winds and high stability encounter a tall mountain range, the low-level flow upstream of the ridge can become blocked, resulting in weak mountain waves and correspondingly minimal tropopause influence (Smith et al. 2002).

Under typical conditions in many mountain ranges, however, $\hat{H}$ is greater than zero and mountain waves strongly modulate the flow field. In this scenario, our results suggest that tropopause height is likely to be just as important as static stability and wind speed in determining the structure of the flow field and, thus, the amount and distribution of orographic precipitation.

While this may be the first paper to assess the influence of the tropopause on orographic precipitation, the ideas presented here owe much to Klemp and Lilly (1975), whose linear explanation for downslope wind storms was based on similar ideas about wave reflection. Their theory has since fallen out of favor, as the essential role of nonlinear dynamics in downslope wind storms has become clearer (Smith 1985; Durran 1986). However, mountain-wave-induced perturbations over the windward slope during precipitation events are much weaker than those in the lee during downslope wind storms, and our results suggest that linear theory can 
provide a very useful framework for understanding the dynamical controls on orographic precipitation.

Acknowledgments. We thank Peter Blossey and David Warren for help configuring the numerical model, Gerard Roe for helpful discussions about FabryPérot interferometers, and three anonymous reviewers for thoughtful comments that improved the manuscript. This work was supported by the National Defense Science and Engineering Graduate Fellowship and by National Science Foundation Grant AGS1138977.

\section{APPENDIX}

\section{Determination of Thermodynamic Quantities $S_{0}$ and $H_{w}$}

In (33), $S_{0}$ represents the condensation per unit vertical displacement at the surface, while $H_{w}$ represents the scale height of moisture. For a saturated air parcel, Siler and Roe (2014) give

$$
S_{0}=\frac{e_{s}}{R_{v}^{2} T_{s}^{2}}\left\{\frac{L_{v} \Gamma_{m}}{T_{s}}\left[\frac{p}{p-(1-\epsilon) e_{s}}\right]-\frac{g}{\epsilon}\right\}
$$

where $e_{s}$ is the saturation vapor pressure, $R_{v}$ is the specific gas constant of water vapor, $T_{s}$ is surface temperature, $L_{v}$ is the latent heat of vaporization, $\Gamma_{m}$ is the moist adiabatic lapse rate, $p$ is pressure, $g$ is the acceleration due to gravity, and $\epsilon$ is the ratio of the gas constants of dry air and water vapor. The linear calculations in sections 3 and 4 were made using $S_{0}=1.9 \times 10^{-3} \mathrm{~g} \mathrm{~m}^{-4}$, which is derived from $p=10^{5} \mathrm{~Pa}, T_{s}=5^{\circ} \mathrm{C}, R_{v}=461 \mathrm{~J} \mathrm{~kg}^{-1} \mathrm{~K}^{-1}, L_{v}=$ $2.5 \times 10^{6} \mathrm{~J} \mathrm{~kg}^{-1}, \epsilon=0.622$, and $\Gamma_{m}=5.8 \mathrm{~K} \mathrm{~km}^{-1}$ [Durran and Klemp 1982, their (19)].

Calculation of $H_{w}$ is less straightforward, since the vertical distribution of moisture is not quite exponential. However, if $H_{w}$ is defined as the $e$-folding height (i.e., the height at which the density of water vapor drops to $1 / e$ times its surface value), then for a saturated atmosphere with $N=0.012 \mathrm{~s}^{-1}$ and a surface temperature of $5^{\circ} \mathrm{C}, H_{w}$ is close to $2.5 \mathrm{~km}$.

SB04's treatment of thermodynamics is essentially the same as ours, but they use different methods to calculate the constants. First, they define the moisturescale height as

$$
H_{w}=\frac{R_{v} T_{s}^{2}}{L_{v} \gamma}
$$

where $\gamma$ is the environmental lapse rate. For the conditions described above, $\gamma \approx 6 \mathrm{~K} \mathrm{~km}^{-1}$, and (A2) yields
$H_{w} \approx 2.4 \mathrm{~km}$, which is not substantially different from the actual $e$-folding value of $2.5 \mathrm{~km}$.

Second, SB04 approximate $S_{0}$ as

$$
S_{0} \approx \frac{C_{w}}{H_{w}}
$$

where $H_{w}$ is the moisture-scale height, and

$$
C_{w}=\frac{e_{s} \Gamma_{m}}{R_{v} T_{s} \gamma}
$$

Together, (A2)-(A4) give

$$
S_{0} \approx \frac{e_{s} L_{v} \Gamma_{m}}{R_{v}^{2} T_{s}^{2}}
$$

which in our case overestimates $S_{0}$ by nearly $50 \%$.

This suggests that while (A2) is quite reasonable, the treatment of thermodynamics within the linear model is significantly improved by replacing (A5) with the exact expression for $S_{0}$ given by (A1).

\section{REFERENCES}

Barcilon, A., and D. Fitzjarrald, 1985: A nonlinear steady model for moist hydrostatic mountain waves. J. Atmos. Sci., 42, 58-67, doi:10.1175/1520-0469(1985)042<0058:ANSMFM>2.0.CO;2.

Barstad, I., and F. Schüller, 2011: An extension of Smith's linear theory of orographic precipitation: Introduction of vertical layers. J. Atmos. Sci., 68, 2695-2709, doi:10.1175/JAS-D-10-05016.1.

Bougeault, P., 1983: A non-reflective upper boundary condition for limited-height hydrostatic models. Mon. Wea. Rev., 111, 420-429, doi:10.1175/1520-0493(1983)111<0420: ANRUBC $>2.0 . \mathrm{CO} ; 2$.

Colle, B. A., 2004: Sensitivity of orographic precipitation to changing ambient conditions and terrain geometries: An idealized modeling perspective. J. Atmos. Sci., 61, 588-606, doi:10.1175/1520-0469(2004)061<0588:SOOPTC $>2.0 . C O ; 2$.

Durran, D. R., 1986: Another look at downslope windstorms. Part I: The development of analogs to supercritical flow in an infinitely deep, continuously stratified fluid. J. Atmos. Sci., 43, 2527-2543, doi:10.1175/1520-0469(1986)043<2527: ALADWP $>2.0 . \mathrm{CO} ; 2$.

_ 2010: Numerical Methods for Fluid Dynamics: With Applications to Geophysics. 2nd ed. Springer, $532 \mathrm{pp}$.

— Väisälä frequency. J. Atmos. Sci., 39, 2152-2158, doi:10.1175/ 1520-0469(1982)039<2152:OTEOMO>2.0.CO;2.

_ and - 1983: A compressible model for the simulation of moist mountain waves. Mon. Wea. Rev., 111, 2341-2361, doi:10.1175/1520-0493(1983)111<2341:ACMFTS>2.0.CO;2.

Eliassen, A., and E. Palm, 1961: On the transfer of energy in stationary mountain waves. Geofys. Publ., 22 (3), 1-23.

Garvert, M. F., B. Smull, and C. Mass, 2007: Multiscale mountain waves influencing a major orographic precipitation event. J. Atmos. Sci., 64, 711-737, doi:10.1175/JAS3876.1.

Houze, R. A., C. N. James, and S. Medina, 2001: Radar observations of precipitation and airflow on the Mediterranean side of 
the Alps: Autumn 1998 and 1999. Quart. J. Roy. Meteor. Soc., 127, 2537-2558, doi:10.1002/qj.49712757804.

Jiang, Q., 2003: Moist dynamics and orographic precipitation. Tellus, 55A, 301-316, doi:10.1034/j.1600-0870.2003.00025.x.

Kessler, E., 1969: On the Distribution and Continuity of Water Substance in Atmosphere Circulations. Meteor. Monogr., No. 32, Amer. Meteor. Soc., 84 pp.

Kirshbaum, D. J., and D. R. Durran, 2004: Factors governing cellular convection in orographic precipitation. J. Atmos. Sci., 61, 682-698, doi:10.1175/1520-0469(2004)061<0682: FGCCIO $>2.0 . \mathrm{CO} ; 2$.

Klemp, J. B., and D. R. Lilly, 1975: The dynamics of wave-induced downslope winds. J. Atmos. Sci., 32, 320-339, doi:10.1175/ 1520-0469(1975)032<0320:TDOWID > 2.0.CO;2.

__ , and D. R. Durran, 1983: An upper boundary condition permitting internal gravity wave radiation in numerical mesoscale models. Mon. Wea. Rev., 111, 430-444, doi:10.1175/ 1520-0493(1983)111<0430:AUBCPI >2.0.CO;2.

Lalas, D. P., and F. Einaudi, 1973: On the stability of a moist atmosphere in the presence of a background wind. J. Atmos. Sci., 30, 795-800, doi:10.1175/1520-0469(1973)030<0795: OTSOAM>2.0.CO;2.

Lilly, D. K., 1962: On the numerical simulation of buoyant convection. Tellus, 14, 148-172.

- 1978: A severe downslope windstorm and aircraft turbulence event induced by a mountain wave. J. Atmos. Sci., 35, 59-77, doi:10.1175/1520-0469(1978)035<0059: ASDWAA $>2.0 . \mathrm{CO} ; 2$.

Medina, S., and R. A. Houze Jr., 2003: Air motions and precipitation growth in Alpine storms. Quart. J. Roy. Meteor. Soc., 129, 345-371, doi:10.1256/qj.02.13.

A. Kumar, and D. Niyogi, 2010: Summer monsoon convection in the Himalayan region: Terrain and land cover effects. Quart. J. Roy. Meteor. Soc., 136, 593-616, doi:10.1002/qj.601.

Neiman, P. J., F. M. Ralph, A. B. White, D. E. Kingsmill, and P. O. G. Persson, 2002: The statistical relationship between upslope flow and rainfall in California's coastal mountains: Observations during CALJET. Mon. Wea. Rev., 130, 1468-1492, doi:10.1175/ 1520-0493(2002)130<1468:TSRBUF $>2.0 . C O ; 2$.

Queney, P., 1960: The airflow over mountains. WMO Tech. Note 34, $135 \mathrm{pp}$.

Rotunno, R., and R. Ferretti, 2001: Mechanisms of intense alpine rainfall. J. Atmos. Sci., 58, 1732-1749, doi:10.1175/ 1520-0469(2001)058<1732:MOIAR > 2.0.CO;2.

_ , and R. A. Houze, 2007: Lessons on orographic precipitation from the Mesoscale Alpine Programme. Quart. J. Roy. Meteor. Soc., 133, 811-830, doi:10.1002/qj.67.

Siler, N., and G. Roe, 2014: How will orographic precipitation respond to surface warming? An idealized thermodynamic perspective. Geophys. Res. Lett., 41, 2606-2613, doi:10.1002/ 2013 GL059095.

,$- \ldots$, and D. Durran, 2013: On the dynamical causes of variability in the rain-shadow effect: A case study of the Washington Cascades. J. Hydrometeor., 14, 122-139, doi:10.1175/ JHM-D-12-045.1.

Smith, R. B., 1979: The influence of mountains on the atmosphere. Advances in Geophysics, Vol. 21, Academic Press, 87-230, doi:10.1016/S0065-2687(08)60262-9.

_ 1985: On severe downslope winds. J. Atmos. Sci., 42, 2597-2603, doi:10.1175/1520-0469(1985)042<2597:OSDW >2.0.CO;2.

_ cipitation. J. Atmos. Sci., 61, 1377-1391, doi:10.1175/ 1520-0469(2004)061<1377:ALTOOP>2.0.CO;2.

, S. Skubis, J. D. Doyle, A. S. Broad, C. Kiemle, and H. Volkert, 2002: Mountain waves over Mont Blanc: Influence of a stagnant boundary layer. J. Atmos. Sci., 59, 2073-2092, doi:10.1175/ 1520-0469(2002)059<2073:MWOMBI >2.0.CO;2.

Wicker, L. J., and W. C. Skamarock, 2002: Time-splitting methods for elastic models using forward time schemes. Mon. Wea. Rev., 130, 2088-2097, doi:10.1175/1520-0493(2002)130<2088: TSMFEM $>2.0 . \mathrm{CO} ; 2$.

Worthington, R. M., and L. Thomas, 1997: Impact of the tropopause on upward propagation of mountain waves. Geophys. Res. Lett., 24, 1071-1074, doi:10.1029/97GL00833. 